\title{
The first spectral line surveys searching for signals from the dark ages
}

\author{
C. M. Persson ${ }^{1}$, R. Maoli ${ }^{2,3}$, P. Encrenaz ${ }^{4}$, A. Hjalmarson ${ }^{1}$, M. Olberg ${ }^{1,5}$, G. Rydbeck ${ }^{1}$, M. Signore ${ }^{4}$, U. Frisk ${ }^{6}$, \\ Aa. Sandqvist ${ }^{7}$, and J. Y. Daniel ${ }^{3}$
}

\author{
1 Onsala Space Observatory (OSO), Chalmers University of Technology, 43992 Onsala, Sweden \\ e-mail: carina.persson@chalmers.se \\ 2 Department of Physics, University of Rome "La Sapienza", Pl. A: Moro 2, 00185, Roma, Italy \\ 3 Institut d'Astrophysique de Paris, 95 bis boulevard Arago, 75014 Paris, France \\ ${ }^{4}$ LERMA, Observatoire de Paris and U.P.M.C., 61, Av. de l'Observatoire, 75014 Paris, France \\ 5 SRON, Landleven 12, 9747 AD Groningen, The Netherlands \\ ${ }^{6}$ Swedish Space Corporation, PO Box 4207, 17104 Solna, Sweden \\ 7 Stockholm Observatory, AlbaNova University Center, 10691 Stockholm, Sweden
}

Received 2 October 2009 / Accepted 8 March 2010

\section{ABSTRACT}

\begin{abstract}
Aims. Our aim is to observationally investigate the cosmic Dark Ages in order to constrain star and structure formation models, as well as the chemical evolution in the early Universe.

Methods. Spectral lines from atoms and molecules in primordial perturbations at high redshifts can give information about the conditions in the early universe before and during the formation of the first stars in addition to the epoch of reionisation. The lines may arise from moving primordial perturbations before the formation of the first stars (resonant scattering lines), or could be thermal absorption or emission lines at lower redshifts. The difficulties in these searches are that the source redshift and evolutionary state, as well as molecular species and transition are unknown, which implies that an observed line can fall within a wide range of frequencies. The lines are also expected to be very weak. Observations from space have the advantages of stability and the lack of atmospheric features which is important in such observations. We have therefore, as a first step in our searches, used the Odin ${ }^{\star}$ satellite to perform two sets of spectral line surveys towards several positions. The first survey covered the band $547-578 \mathrm{GHz}$ towards two positions, and the second one covered the bands $542.0-547.5 \mathrm{GHz}$ and $486.5-492.0 \mathrm{GHz}$ towards six positions selected to test different sizes of the primordial clouds. Two deep searches centred at 543.250 and $543.100 \mathrm{GHz}$ with $1 \mathrm{GHz}$ bandwidth were also performed towards one position. The two lowest rotational transitions of $\mathrm{H}_{2}$ will be redshifted to these frequencies from $z \sim 20-30$, which is the predicted epoch of the first star formation.

Results. No lines are detected at an rms level of 14-90 and 5-35 mK for the two surveys, respectively, and 2-7 mK in the deep searches with a channel spacing of 1-16 MHz. The broad bandwidth covered allows a wide range of redshifts to be explored for a number of atomic and molecular species and transitions. From the theoretical side, our sensitivity analysis show that the largest possible amplitudes of the resonant lines are about $1 \mathrm{mK}$ at frequencies $\$ 200 \mathrm{GHz}$, and a few $\mu \mathrm{K}$ around $500-600 \mathrm{GHz}$, assuming optically thick lines and no beam-dilution. However, if existing, thermal absorption lines have the potential to be orders of magnitude stronger than the resonant lines. We make a simple estimation of the sizes and masses of the primordial perturbations at their turnaround epochs, which previously has been identified as the most favourable epoch for a detection. This work may be considered as an important pilot study for our forthcoming observations with the Herschel Space Observatory.
\end{abstract}

Key words. cosmology: observations - early Universe - large-scale structure of Universe - line: formation - ISM: molecules submillimeter: ISM

\section{Introduction}

One of the important topics in astronomy today concerns the formation of the first stars and structure formation in the Universe. The cosmic microwave background radiation observed by NASAs Cosmic Background Explorer ${ }^{1}$ (COBE) satellite and the Wilkinson Microwave Anisotropy Probe ${ }^{1}$ (WMAP)

* Odin is a Swedish-led satellite project funded jointly by the Swedish National Space Board (SNSB), the Canadian Space Agency (CSA), the National Technology Agency of Finland (Tekes) and Centre National d'Etudes Spatiales (CNES). The Swedish Space Corporation was the prime contractor and also is responsible for the satellite operation.

${ }^{1}$ http://lambda.gsfc.nasa.gov/ shows small density fluctuations at a redshift of $z \approx 1100$ and a temperature of $\approx 3000 \mathrm{~K}$. After this epoch of recombination the Universe became neutral and entered the cosmic Dark Ages which did not end until the formation of the first stars and quasars, which are believed to have reionised the Universe at $z \sim 11$ (Komatsu et al. 2009; Dunkley et al. 2009). During the Dark Ages the transition from the small density fluctuations left over from the inflation to completely formed objects took place (e.g. Loeb 2008). The challenging question is how and by what means we can reveal this process.

The main method to obtain information about physical and chemical conditions in star forming regions at low redshift is by means of atomic and molecular line observations. Molecular observations of high redshift objects have also been performed, for 
example by Bertoldi et al. (2003) who found high excitation CO in a Sloan Digital Sky Survey quasar at $z=6.4$. However, no observational evidence exists at all from the cosmic Dark Ages at even higher redshifts before or during the earliest epoch of the first star formation at predicted redshifts of $\sim 20-30$ (e.g. Glover 2005; Glover et al. 2008).

Model-dependent theoretical analysis and computer simulations have therefore been the main tools to follow perturbations of different scales in the primordial medium to predict when and how the first stars and structure in the Universe evolved. This depends on a number of parameters including the chemistry in the Early Universe and the properties of dark matter. The chemistry constitutes a very important part in the Early Universe since star formation requires a cooling mechanism mainly provided by molecules. Dust grains are very important for molecular production and for instance almost all of the molecular hydrogen production occurs today on the surface of dust grains. The lack of dust and heavy elements in the Early Universe has therefore resulted in very low molecular abundances of only a few species and therefore difficulties to explain the formation of the first stars (e.g. Bromm \& Larson 2004; Glover 2005).

The Standard Big Bang Nucleosynthesis (SBBN) model predicts the formation of $\mathrm{H}, \mathrm{D}, \mathrm{He}$ and $\mathrm{Li}$ a few minutes after the Big Bang (e.g. Steigman 2007). Their primordial abundances in this model depend only on the density of baryons which has been measured with high precision, $\Omega_{\mathrm{b}}=0.0456 \pm 0.0015$ (Komatsu et al. 2009). In the expanding and cooling Universe, molecular synthesis of for example $\mathrm{H}_{2}, \mathrm{H}_{2}^{+}, \mathrm{HeH}^{+}, \mathrm{HD}, \mathrm{HD}^{+}, \mathrm{LiH}$, and $\mathrm{LiH}^{+}$could begin as soon as neutral atoms appeared, attaining maximum abundances around redshifts of 100-400 depending on species (e.g. Vonlanthen et al. 2009). The molecular primordial abundances depend on many parameters and many attempts have been made to follow the chemical evolution during the Dark Ages with results that often differ by orders of magnitude (e.g. Lepp \& Shull 1984; Puy et al. 1993; Galli \& Palla 1998, 2002; Lepp et al. 2002; Black 2006; Puy \& Signore 2007; Schleicher et al. 2008; Sethi et al. 2008; Signore \& Puy 2009; Bovino et al. 2009).

The behaviour of gas in galaxy formation is still an open problem, despite the development of simulations, more precise semi-analytical models and more complete observational data during the recent years. In particular, cooling mechanisms in the primordial medium, star formation and feedback processes are far from being completely understood (Ciardi \& Ferrara 2005; Baugh 2006; Ellis \& Silk 2007; Okamoto 2008; De Lucia \& Poggianti 2008). In order to be able to discriminate between the different models of structure formation it is therefore important to test theory with observations from the Dark Ages.

We do know that during this epoch, the Universe is filled with cosmic microwave background (CMB) photons, atoms, molecules, ions and electrons. The question is if these species have high enough abundances to produce detectable signals. Since the average density on large scales is predicted to be very low, $n_{\mathrm{H}} \sim 0.2 \mathrm{~cm}^{-3}$ at $z \sim 100$ and decreasing towards lower redshift, collisions are negligible and excitation is generally believed to be dominated by absorption of CMB photons followed by spontaneous or stimulated emission in a process known as resonant scattering. This process alone is not able to produce a signal since CMB photons are nearly isotropic and resonant scattering produce an isotropic distribution of photons. The only effect of resonant scattering by a primordial medium is the damping of CMB primary anisotropies (Maoli et al. 1994). However, the gravitational potentials induce peculiar velocities of the primordial clouds. Thus, the scattered CMB photons will be isotropically distributed in the reference frames of the clouds, but not in the CMB reference frame. As a consequence, secondary resonant scattering anisotropies will be produced. The cross section for the resonant scattering is many orders of magnitudes larger than for Thomson scattering between CMB photons and electrons, but it is extremely frequency dependent, peaking at the rotational and rotational-vibrational frequencies of the scattering species. Resonant lines will therefore be produced by these moving primordial clouds (Maoli et al. 1996).

Since the original idea by Dubrovich (1977), inspired by earlier work on CMB fluctuations by Zeldovich, a number of papers have already analysed the possibility of detecting the resonant lines (e.g. Maoli et al. 1994, 1996; Dubrovich et al. 2008) and two previous observational attempts have been made without success (de Bernardis et al. 1993; Gosachinskij et al. 2002).

However, possible signals from the Dark Ages may not only arise from resonant scattering since the growing potentials may further induce adiabatic heating as well as shock heating of collapsing primordial perturbations (e.g. Barkana \& Loeb 2001; Santoro \& Shull 2006; Johnson \& Bromm 2006; Greif et al. 2008 b). This produces thermal emission or absorption from denser than average or high temperature regions. The first stars are predicted to be very massive $\gtrsim 100 M_{\odot}$ and formed either in isolation or at most as a small stellar multiple in minihalos (e.g. Abel et al. 2000; Bromm \& Larson 2004; Bromm et al. 2009). These hot stars must have emitted enormous amounts of energetic radiation. By the time of reionisation, large HII regions are predicted to have grown around clusters of these hot and bright stars (e.g. Greif et al. 2008a). This radiation will first destroy the molecules, but in the cooling ejecta of the primordial supernovae molecules will later be efficiently produced (Cherchneff \& Lilly 2008) for instance $\mathrm{H}_{2}$ due to the high degree of ionisation. However, many considerations have to be taken into account to predict all these types of emission/absorption, hence leading to uncertain results.

We emphasize that every possibility to obtain observations from the Dark Ages should be seriously considered. A detection would be extremely important and would give direct evidence of a very high redshift protostructure within a wide range of angular sizes, from arcsecond to arcminute scales, depending on the cosmological model. These small scales are not observable in the CMB anisotropies due to the finite depth of the last scattering surface. The horizon at the last scattering surface corresponds to $240 \mathrm{Mpc}$ today. The Planck ${ }^{2}$ satellite, launched on May 14, 2009, will measure the CMB power spectrum for angular scales greater than $10^{\prime}(40 \mathrm{Mpc})$. Smaller scales, at cluster and galaxy sizes are unobservable due to photon diffusion damping associated with the non-instantaneous nature of the recombination process at the last scattering surface (Silk 1968).

Any primordial spectral line will also provide a unique test of nuclear synthesis at high redshift, give new information about the reionisation of the universe, probe the chemistry throughout a wide range of redshifts, the heating and cooling processes as well as the dynamics of the primordial clouds before and during the gravitational collapse of a protostructure. Even a nondetection could give very valuable information in order to constrain all these issues if the noise level is low.

Given the expected weakness of the lines and our ignorance of the frequencies at which the transitions will fall, groundbased observations are complicated by the terrestrial atmospheric lines. The ozone molecule, $\mathrm{O}_{3}$, including all the isotopes ${ }^{16} \mathrm{O},{ }^{17} \mathrm{O}$ and ${ }^{18} \mathrm{O}$, emits numerous lines. Moreover, most

\footnotetext{
${ }^{2}$ http://planck.esa.int
} 
of the vibrationally excited lines can not be found in catalogues. Observations with the IRAM 30-m telescope (de Bernardis et al. 1993) experienced difficulties from these lines at a low level. Interferometer observations are therefore interesting for searches like this, due to the many advantages for rejecting both broad and narrow band atmospheric emissions. Ground based single dish observations can, however, also provide valuable information especially at low frequencies, even though the foreground and atmospheric radiation poses a large problem. Another possibility is observations from space, which have the advantages of stability and lack of atmospheric features.

As a first test, we have therefore chosen to use a satellite to search for and put upper limits on primordial signals from the Dark Ages. The satellite is required to have tunable receivers in order to cover a broad spectral band, essential for the exploration of a wide redshift range. In this work, we have used the only available satellite at the time with the above mentioned requirements: the Odin satellite (Nordh et al. 2003). This satellite has the unique capability to cover a broad spectral band with a high channel spacing of about $1 \mathrm{MHz}$ using tunable single sideband (SSB) receivers. This has allowed us for the first time to search for redshifted molecular hydrogen from the predicted time of the formation of the first stars or earlier.

\section{The Odin observations}

The main problems in the searches of primordial lines are the unknown but expected weak amplitudes from unknown species in unknown sources at unknown redshifts. This implies that the frequency of the lines can be anywhere in a wide frequency range. Thus we need to cover a large frequency band to probe a wide range of redshifts for many possible species and transitions. The sources should on the other hand be found in every direction on the sky even though they most likely have a clumpy distribution and also are located at different redshifts. This implies that we do not know the evolutionary state, size or density of the perturbations.

\subsection{Interesting species}

Our approach in all observations has been to perform spectral line surveys in order to cover a wide redshift range. This is necessary since our objects are located at an unknown redshift and the expansion of the Universe causes the frequency of an emitted photon to be redshifted as

$$
\frac{v_{0}}{v_{\mathrm{obs}}}=1+z
$$

where $v_{0}$ is the rest frequency, $v_{\mathrm{obs}}$ the observed frequency of the transition, and $z$ is the redshift. When we choose an observation frequency, we thus determine the emitting redshift for each transition given by Eq. (1). Each transition may also be detected at different frequencies, emitted from several objects at different redshifts along the line of sight. The strong frequency dependence of the scattering and emission processes gives rise to lines superposed with the black body continuum spectrum of the CMB. Each line is associated with a moving primordial perturbation, exactly in the same way the Lyman $\alpha$ absorption lines are associated with neutral hydrogen clouds which absorb the light from bright quasars and galaxies.

We search for any atom or molecule that may be present in the high redshift Universe. This includes molecules that form from the primordial elements H, D, He and Li (Steigman 2007) such as: $\mathrm{H}_{2}, \mathrm{H}_{2}^{+}, \mathrm{H}_{2} \mathrm{D}^{+}, \mathrm{HD}, \mathrm{HD}^{+}, \mathrm{HeH}^{+}, \mathrm{LiH}$ and $\mathrm{LiH}^{+}$. There are also suggestions that lines from neutral hydrogen $\mathrm{H}$ and helium He could be seen during the time of respective recombination and at later times (Rubiño-Martín et al. 2006; Chluba \& Sunyaev 2006; Sethi et al. 2007; Chluba et al. 2007; Rubiño-Martín et al. 2008). Prior to the reionisation at $z \gtrsim 6$ the universe was, however, opaque to for instance $\mathrm{Ly} \alpha$ radiation (Becker et al. 2001).

Previous searches for primordial resonant lines were mainly focused on $\mathrm{LiH}$ due to its high dipole moment and a possibly high abundance (de Bernardis et al. 1990). New ab initio calculations of reaction rates of $\mathrm{LiH}$ destruction in the early universe show, however, that the $\mathrm{LiH}$ abundance is too low to allow a detection (Bovino et al. 2009). Another interesting molecule with a high dipole moment is $\mathrm{HeH}^{+}$which, together with a much higher abundance than $\mathrm{LiH}$, increases the possibility of a detection.

Moreover, non-standard BBN models predict heavy nuclei such as carbon, oxygen, nitrogen, and flourine, which may have created molecules like $\mathrm{CH}, \mathrm{OH}, \mathrm{NH}, \mathrm{HF}$, and their respective molecular ions (Puy et al. 2007; Campos et al. 2007; Vonlanthen \& Puy 2008; Vonlanthen et al. 2009). For example the abundance of the $\mathrm{CH}$ molecule is found to be higher than the $\mathrm{H}_{2} \mathrm{D}$ or $\mathrm{HD}$ abundances in the inhomogeneous non-standard BBN models. In effect, a number of possible mechanisms have been suggested to generate density perturbations in the early universe which could survive until the onset of primordial nucleosynthesis. Such inhomogeneities in the baryon number may result from non-equilibrium processes in the Big Bang, for example, occurring during a putative first-order quantum-chronodynamics (QCD) phase transition at $\sim 100 \mathrm{MeV}$ or during the electroweak symmetry breaking at $\sim 100 \mathrm{GeV}$. Assuming the existence of such surviving density fluctuations, some authors have shown that - in the framework of these non-standard BBN scenarios the altered primordial nucleosynthesis could lead to the synthesis of heavy elements (e.g. Applegate et al. 1987; Rauscher et al. 1994; Kurki-Suonio et al. 1997; Lara et al. 2006). Note also that these models satisfy the observed primordial elemental light abundances.

In addition, regardless of BBN model, the first generation stars of a primordial composition formed at $z \gtrsim 20-30$ might quickly have polluted the medium with small amounts of metals with subsequent chemistry (e.g. Mackey et al. 2003; Bromm \& Larson 2004; Cherchneff \& Dwek 2009; Smith et al. 2009). Several species have already been observed at high redshifts. In addition to the high excitation CO observations at $z=6.4$ (Bertoldi et al. 2003) this molecule has also been observed in for example a damped Lyman- $\alpha$ system at $z=2.4$ (Srianand et al. 2008) and in a field containing an over-density of Lyman break galaxies at $z=5.1$ (Stanway et al. 2008). A massive CO reservoir has also been detected at $z=3.9$ (Papadopoulos et al. 2001). An unusually high amount of neutral hydrogen was found by Frye et al. (2008) in a $14 \mathrm{Mpc}$ region surrounding a young galaxy at $z=4$.9. A strong detection of molecular absorption bands, including $\mathrm{H}_{2}$ and $\mathrm{CO}$, was also recently observed from gas within the host galaxy of the gamma-ray burst 080607 at $z=3.0363$ (Prochaska et al. 2009). Several absorption features are still unidentified, but a $\mathrm{H}_{2}$ column density of $10^{21.2 \pm 0.2} \mathrm{~cm}^{-2}$ was inferred with an excitation temperature of $10-300 \mathrm{~K}$. The highest redshift object observed today is the gamma-ray burst of 23 April 2009 at $z=8.2$ (Tanvir et al. 2009; Salvaterra et al. 2009). Some long duration gamma-ray bursts can be primordial supernovaes originating from the super-massive first generation stars, i.e. population III stars (Woosley \& Bloom 2006). 
The continuum of $\mathrm{H}^{-}$has also been suggested to give rise to a detectable signal in terms of a decrease in the CMB spectrum which may superimpose a detectable absorption feature on the CMB. This could be detectable with the Planck satellite, although the strength of this effect has been estimated to quite different values (Black 2006; Schleicher et al. 2008).

Besides producing molecular lines, resonant scattering has an additional interesting feature that is regardless of peculiar velocities, this process has the potential of reducing the power of the CMB primary anisotropies if the molecular abundances are high enough (Maoli et al. 1994). Planck may be able to detect also this decrease in power. There are also several suggestions to use differential measurements of the broad band CMB angular power spectrum observed with Planck in the search for resonant lines (e.g. Basu et al. 2004; Dubrovich et al. 2008; Schleicher et al. 2008). To reach the required sensitivity of the order of $\mu \mathrm{K}$, the main problem to extract these weak signals with this approach, will be the galactic and extra-galactic foreground emissions.

Since the most abundant species is neutral hydrogen, a very interesting and promising transition is the $21 \mathrm{~cm}$ line. Such difficult observations must, however, await the future Lowfrequency $\operatorname{Array}^{3}$ (LOFAR) and the proposed Square Kilometer Array (SKA) ${ }^{4}$ which will be able to detect the redshifted $21-\mathrm{cm}$ HI transition between $z \sim 6-11$ or at even higher redshifts (e.g. Hogan \& Rees 1979; Furlanetto et al. 2009). Both instruments, however, face severe difficulties when subtracting the astrophysical foreground contamination which is several orders of magnitudes stronger than the $21 \mathrm{~cm}$ line (Bowman et al. 2009).

Most other atomic transitions than the $21 \mathrm{~cm}$ line have much higher required excitation temperatures than the molecular transitions and, except for $\mathrm{H}, \mathrm{He}$ and $\mathrm{Li}$, must in addition have been produced either by the first stars or in a non-standard BBN. They are therefore only expected to be seen at redshifts during the epoch of first star formation or some time after. As a first step, we have therefore concentrated our searches on optically thin primordial molecular lines. The redshifted frequencies from $z \sim 20-30$ of the lowest transitions of ortho- and para $-\mathrm{H}_{2}$ fall at Odin frequencies around $500 \mathrm{GHz}$. These lines could be observable in absorption towards for example hot HII regions. The detectability of $\mathrm{H}_{2}$ rotational lines in emission associated with the formation of the first stars has previously been discussed (Omukai \& Kitayama 2003; Kamaya \& Silk 2003) in addition to the ro-vibrational $\mathrm{H}_{2}$ emission lines (Mizusawa et al. 2004). In addition, a number of lines of for example $\mathrm{H}_{2}^{+}, \mathrm{H}_{2} \mathrm{D}^{+}, \mathrm{HD}$, $\mathrm{HD}^{+}$, and $\mathrm{HeH}^{+}$can also be seen from this epoch.

At redshifts higher than about $z \gtrsim 150-200$ the kinetic matter temperature is expected to be the same as the radiation temperature of the $\mathrm{CMB}$ due to Compton scattering, evolving as

$T_{\mathrm{CMB}}=T_{\mathrm{K}}=T_{0}(1+z)$,

where $T_{0}=2.725 \mathrm{~K}$ is the cosmic microwave background radiation $(\mathrm{CMB})$ today, as measured by the FIRAS instrument onboard the COBE satellite (Fixsen et al. 1996). During this epoch only resonant scattering may occur since temperature differences are required to produce thermal spectral lines (see Eq. (23)). At redshifts $\lesssim 200$, the matter temperature evolves following an adiabatic expansion which implies a kinetic temperature decrease faster than for the CMB (Glover 2005)

$T_{\mathrm{K}} \propto(1+z)^{2}$.

\footnotetext{
3 http://www. lofar.org/

4 http://wWW. skatelescope.org/
}

These temperature differences will force the excitation further away from equilibrium with the radiation, and should enhance spectral absorption distortions at redshifts $\$ 150-200$. The gas will eventually be heated by the accretion and collapse phases of the growing perturbations, and eventually by the first stars and quasars which will reionise the whole universe at $z \gtrsim 6$. This will enhance spectral emission features.

The coupling of the excitation temperature to the radiation or matter temperature differs with species. Polar molecules like $\mathrm{HeH}^{+}$probably continue to have $T_{\mathrm{ex}}$ coupled to the CMB temperature, while $\mathrm{H}_{2}$ may remain more strongly coupled to the matter temperature. There are also situations where the excitation temperature may depend on molecular formation processes, by which for instance primordial $\mathrm{H}_{2}$ may remain superthermal relative to the matter temperature.

The strength of a spectral line depends, in addition to the number density and dipole moment, on the ratio of the upper state energy (in Kelvin) and excitation temperature (see Eq. (13)). Rotational-vibrational lines, with upper state energies of thousands of Kelvin, are therefore only expected for very high redshift sources with a high temperature or from collapsing high-temperature regions at lower redshifts. In such regions the density may be increased above average, and the increased collision rate in these regions will drive the level populations and the excitation temperature towards the high matter temperature.

A detection of several spectral lines is required to secure a molecular identification and a redshift of an emitting source. The spectral distance between rotational lines is, however, rather wide, even though it becomes increasingly more narrow for highly redshifted sources observed below 100-200 GHz. It is thus more likely that a single transition from each object would be seen in the Odin observations. The rotational-vibrational transitions would be more closely spaced, but have a lower probability of detection.

\subsection{Observational strategy}

Neither the amplitude nor the line widths are known and therefore we use high spectral resolution to enable detection of narrow lines, and later re-bin the data to lower resolution. The choice of spectral resolution affects the root-mean-square noise level $T_{\text {rms }}$ as can be seen from the radiometer formula

$$
\frac{T_{\mathrm{rms}}}{T_{\mathrm{sys}}}=\frac{K}{\sqrt{t \Delta f}},
$$

where $\Delta f$ is the frequency resolution of the spectrometer, $t$ is the on-source integration time, $T_{\text {sys }}$ is the noise temperature from the whole system, which includes the receiver noise, and $K$ is a constant which depends on the observation strategy $(K=\sqrt{2}$ for a switched receiver).

The expected signals most likely originate from every direction in the sky, even though they will be stronger from rare high density peaks. However, we needed to observe positions with as low contamination as possible from any known source, and in addition they had to be observable with the Odin satellite for long periods. For these reasons we have observed towards two of the WMAP hot spots in the CMB radiation, out of the Galactic plane. The coordinates are listed in Table 1.

Two different sets of observations were performed with different observation strategies as described below. The first survey took place in 2004 and the second one in 2006-2007. 
C. M. Persson et al.: The first spectral line surveys searching for signals from the dark ages

Table 1. Coordinates of observations.

\begin{tabular}{lll}
\hline \hline Position & RA $(J 2000)$ & $\operatorname{Dec}(J 2000)$ \\
\hline Hot Spot 1 & $05^{\mathrm{h}} 26^{\mathrm{m}} 00^{\mathrm{s}} 0$ & $-48^{\circ} .30^{\prime} 00^{\prime} 0$ \\
Hot Spot 2 & $05^{\mathrm{h}} 09^{\mathrm{m}} 36^{\mathrm{s}} 0$ & $-43.24^{\prime} .0 \prime^{\prime} 0$ \\
\hline
\end{tabular}

In addition, we performed two sets of deep searches at one frequency setting towards one position in 2007 and 2009.

The Odin $1.1 \mathrm{~m}$ offset Gregorian telescope has a circular beam at $557 \mathrm{GHz}$ and a FWHM beam width of 2!1 (Frisk et al. 2003). This corresponds for example to 540 and $78 \mathrm{kpc}$ at $z=10$ and $z=100$, respectively (see also Table C.2, on-line material). Being outside the atmosphere, and with an exceptionally high main beam efficiency, $\eta_{\mathrm{mb}}=0.9$, our intensity calibration is very accurate. The calibration procedure (the chopper wheel method) of the Odin satellite is described in Olberg et al. (2003). The intensity scale in the figures is expressed in terms of antenna temperature $T_{\mathrm{A}}^{*}$. The reconstructed pointing offset was $\lesssim 15^{\prime \prime}$ during most of the time.

Two different single sideband (SSB) receivers, with a typical side-band suppression of $\gtrsim 20 \mathrm{~dB}$, were used simultaneously in combination with one auto-correlator (AC) and one acoustooptical spectrometer (AOS). The bandwidth is $1.040 \mathrm{GHz}$ for the $\mathrm{AOS}$, and $690 \mathrm{MHz}$ for the $\mathrm{AC}$, with a channel spacing of 0.62 and $1.0 \mathrm{MHz}$ for the AOS and AC, respectively, and thus $\Delta v / v \approx 2 \times 10^{-6}$ at $v=545 \mathrm{GHz}$. The Odin average system temperature is around $3300 \mathrm{~K}$ (SSB) and therefore many hours of observations are demanded to produce sensitive observations. The resulting noise levels may very well be too high to allow a detection, but the intensity of the lines is really not known and we have therefore taken a pure observational approach. If no detection is obtained, the resulting upper limits will be used as input to the noise levels required in future observations. This approach also applies to the chosen frequency resolution which is connected to the noise level and final covered bandwidth.

In each Local Oscillator (LO) setting, the resulting AOS spectra have a very stable baseline except at the band edges where the calibration spectra exhibit a steep increase. The noisy edges are excluded, and then a first or second order polynomial baseline is subtracted before we join all spectra together to form a contiguous spectrum. The AC consists of 7 bands of $100 \mathrm{MHz}$ each in each setting. We subtract a linear baseline in each band before we align and average all spectra. All averages are then joined.

\subsubsection{Observations during summer 2004}

Our first goal was to cover as wide a frequency band as possible. We were awarded 337 orbits ( 1 orbit $\sim 1$ h of observation) and performed independent position switching observations towards the two positions listed in Table 1, using reference positions offset by -45 arcmin in declination. Using simultaneous observations of the AOS and AC, $15.5 \mathrm{GHz}$ were covered in steps with each receiver: $\mathrm{AC} \sim 563-578 \mathrm{GHz}$ and $\mathrm{AOS} \sim 547-563 \mathrm{GHz}$, giving at total observed frequency band of $31 \mathrm{GHz}$ covering the full 547-578 GHz band. We spent 5 orbits for each LO setting and the step size in frequency was $0.5 \mathrm{GHz}$. The settings and steps were the same as in the first spectral line survey performed by the Odin satellite towards the Orion KL nebulae (Olofsson et al. 2007; Persson et al. 2007).

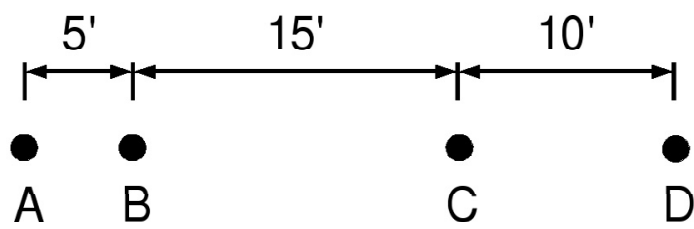

Fig. 1. Observation strategy during the second spectral survey 2006/07 (also described in Table 2). The Odin beam has a FWHM width of 2'.1.

Table 2. Observation strategy 2006/07.

\begin{tabular}{llll}
\hline \hline & Sig & Ref. & Spacing \\
\hline 1 & A & B & $5^{\prime}$ \\
2 & B & C & $15^{\prime}$ \\
3 & C & D & $10^{\prime}$ \\
4 & D & A & $30^{\prime}$ \\
\hline
\end{tabular}

Notes. Every weekend we observed four positions, A-D, in a sequence using $\sim 10 \mathrm{~h}$ towards each position with one frequency setting (also shown in Fig. 1).

\subsubsection{Observations during $2006 / 07$}

In the next attempt during nine weekends in winter 2006/07 and one weekend in Aug. 2007 (in total 464 orbits) we changed our observational strategy. We wanted to lower the noise compared to what we obtained in the 2004 observations, but still needed to cover a wide frequency band. Since the sizes of the primordial objects are unknown, this time we also wanted to test different spacings between the signal and reference positions in order to avoid subtraction of the signal if present in both positions. A compromise of the above requirements resulted in the scheme shown in Fig. 1. Four positions, A-D, were observed where position A is the previously Hot Spot 1 observed during 2004. The minimum offset was determined by the Odin beam size of 2.1 , and thus we chose the following offsets: $-5^{\prime},-15^{\prime}$, and $-10^{\prime}$ in Dec, keeping the same RA During 10 weekends of observations ( $\sim 40$ orbits/weekend) we observed all four positions with one frequency setting each weekend. Table 2 describes how we performed the observations: 10 orbits towards each pair of positions were observed in a sequence. First towards position $A$ with $B$ as reference $(A B)$, then $B$ with $C$ as a reference (BC), $\mathrm{C}$ with $\mathrm{D}$ as reference $(\mathrm{CD})$, and finally $\mathrm{D}$ with $\mathrm{A}$ as a reference (DA). Thus, in total we have ten orbits/setting/position for ten different LO settings.

As in the first observation run in 2004, we performed simultaneous observations with the AC and the AOS, but covering other frequency ranges. The final results are spectral surveys in the frequency ranges $542.0-547.5 \mathrm{GHz}$ (AOS) and 486.5-492.0 GHz (AC) towards four positions.

\subsection{Deep searches at $543 \mathrm{GHz}$ during 2007 and 2009}

In addition to the spectral surveys we also wanted to perform a deep search to lower the noise even further. In 2007 we therefore used 40 orbits towards HotSpot1 position A with B as reference position with $543.250 \mathrm{GHz}$ as centre frequency with the AOS and $490.250 \mathrm{GHz}$ with the AC. This produced an rms level of about $10 \mathrm{mK}$ over the $1 \mathrm{GHz}$ band with a channel spacing of $1 \mathrm{MHz}$, and a possible $4 \sigma$ detection at $543.1 \mathrm{GHz}$. To validate the possible detection, we performed additional observations in April 2009 using 96 orbits and then changed the centre frequency of the AOS to be $543.1 \mathrm{GHz}$. 


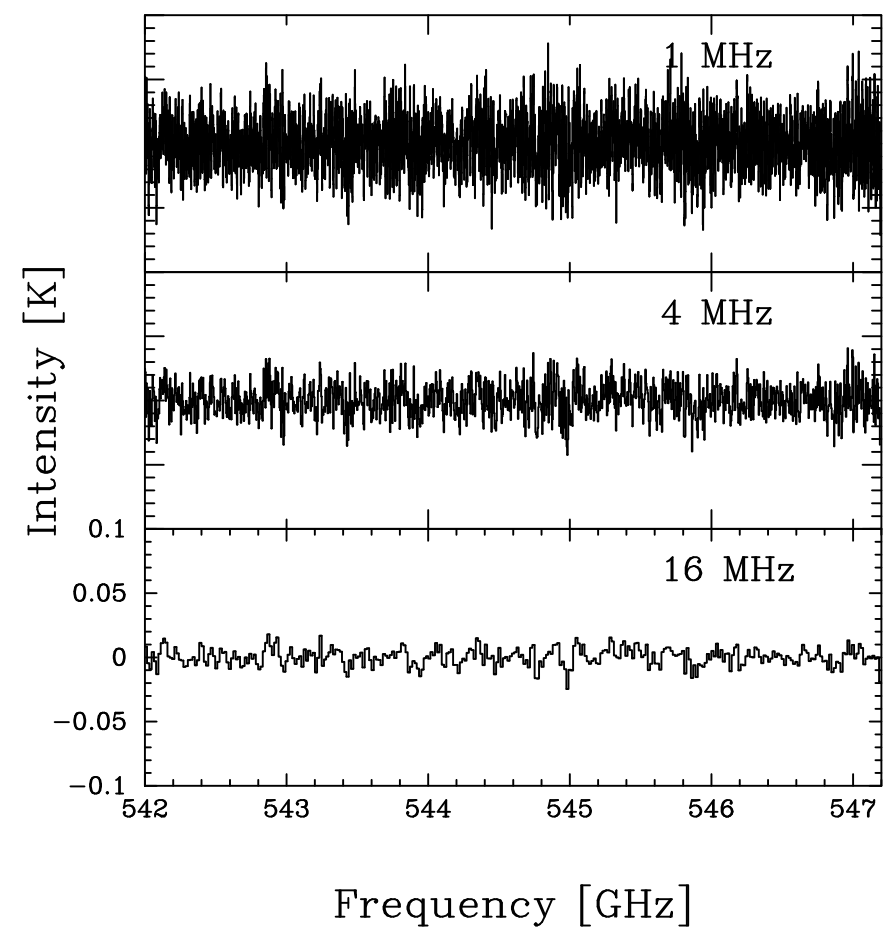

Fig. 2. Examples of the resulting spectra: 2006/07 observations towards Hot Spot 1 (position A with position B used as reference) is shown with three different channel spacings: 1,4 , and $16 \mathrm{MHz}$ with corresponding $1 \sigma$ noise levels of $20,13,7 \mathrm{mK}$.

\section{Results}

No lines were detected and we are thus limited to set upper limits of possible signals. We choose a $5 \sigma$ noise level as an upper limit since the peak noise level is always lower than this value if the noise follows a Gaussian distribution and has independent velocity channels. In this case, the probability that an observed $5 \sigma$ line would be a noise feature is less than $10^{-6}$.

The noise is rather stable over the covered band of all surveys, with a few exceptions where we had loss of observation time. We therefore measure the $1 \sigma$ noise level across the total band covered, towards each position. We have re-binned the AOS data to the AC channel spacing of $1 \mathrm{MHz}$, and then rebinned both AOS and AC to channel spacings of 4 and $16 \mathrm{MHz}$, with an example shown in Fig. 2. This rebinning should lower the noise by a factor of 2 and 4, respectively, if the noise is Gaussian distributed. However, our measurements do suffer from baselines at a low level which prevents the noise to follow Eq. (4) exactly. All $1 \sigma$ results, except the deep searches, are found in Tables 3 and 5, which also include the peak intensity of the noise given in terms of the measured $\sigma$.

Figures A.1 and A.2 (on-line material) show the original AOS spectra used to produce Fig. 2. The intensity is plotted vs. channel numbers and shown for all ten frequency settings.

Note, that we do not know if the signals we seek are absorption or emission lines, or if they are located in the signal or the reference position.

\subsection{Comments on results from the 2004 observations}

The observations from summer 2004 resulted in a wide frequency band, but with a rather high noise level. In Table 3 we see that a typical $1 \sigma$ is about $38(85), 23(45)$, and $15(26) \mathrm{mK}$
Table 3. Upper limits from observations during 2004 using 1, 4 and $16 \mathrm{MHz}$ channel spacings.

\begin{tabular}{ccc}
\hline \hline \multicolumn{3}{c}{$1 \mathrm{MHz}$ channel spacing } \\
$1 \sigma$ & Peak Intensity \\
Position & {$[\mathrm{mK}]$} & {$[\sigma]$} \\
& $37(88)$ & $3.8(4.0)$ \\
\hline HotSpot 1 & $40(83)$ & $3.6(3.9)$ \\
\hline \multicolumn{3}{c}{$4 \mathrm{MHz}$ channel spacing } \\
HotSpot 2 & $22(46)$ & $3.1(3.3)$ \\
HotSpot 2 & $24(44)$ & $3.2(3.4)$ \\
\hline \multicolumn{3}{c}{$16 \mathrm{MHz}$ channel spacing } \\
HotSpot 1 & $14(27)$ & $2.9(3.1)$ \\
HotSpot 2 & $13(25)$ & $3.0(3.2)$ \\
\hline
\end{tabular}

Notes. The limits are for the data observed with the AOS(AC) and for frequencies between 547-563 (563-578) GHz.

Table 4. Observational strategy 2006/07.

\begin{tabular}{ccccc}
\hline \hline Position & \multicolumn{4}{c}{ Angular sensitivity $\theta$} \\
& $2-5^{\prime}$ & $5-10^{\prime}$ & $10-15^{\prime}$ & $15-30^{\prime}$ \\
\hline $\mathrm{A}$ & $\mathrm{AB}+\mathrm{AD}$ & $\mathrm{AD}$ & $\mathrm{AD}$ & $\mathrm{AD}$ \\
$\mathrm{B}$ & $\mathrm{BC}+\mathrm{BA}$ & $\mathrm{BC}$ & $\mathrm{BC}$ & - \\
$\mathrm{C}$ & $\mathrm{CD}+\mathrm{CB}$ & $\mathrm{CD}+\mathrm{CB}$ & $\mathrm{CB}$ & - \\
$\mathrm{D}$ & $\mathrm{DA}+\mathrm{DC}$ & $\mathrm{DA}+\mathrm{DC}$ & $\mathrm{DA}$ & $\mathrm{DA}$ \\
\hline
\end{tabular}

Notes. Different combinations of signal and reference positions are sensitive to different angular sizes of the primordial perturbations.

for the $\operatorname{AOS}(\mathrm{AC})$ observations with 1, 4 and $16 \mathrm{MHz}$ channel spacing, respectively.

\subsection{Comments on results from the $2006 / 07$ observations}

Due to the new scheme the analysis of these observations is somewhat different from that of the 2004 observations. As seen from Fig. 1, each pair of observations is sensitive to different angular scales of the perturbations. As an example, if we assume that the signal is in position A and use B as reference, this combination is sensitive to a perturbation size between $2-5^{\prime}$. If the primordial cloud is larger than $5^{\prime}$ the signal will be present in both $\mathrm{A}$ and $\mathrm{B}$ and will disappear in the resulting $\mathrm{AB}$ average. We are, however, also sensitive to larger sizes of the perturbations in A since we have observations towards position D with A as a reference. If we switch and use A as signal and D as reference, we will have an $\mathrm{AD}$ average which is sensitive to scales between $2-30^{\prime}$. Therefore, we can average both $\mathrm{AB}$ and $\mathrm{AD}$ together to further lower the noise with a sensitivity of scales $2-5^{\prime}$. For larger scales we can only use AD. A summary of all average combinations for each position with corresponding angular scale sensitivity is given in Table 4.

This observation strategy is also a way to check if the signal is in the signal or reference position. For example, if there is a signal in position $\mathrm{A}$, and the size of the perturbation is $2-5^{\prime}$, the signal-to-noise $(\mathrm{S} / \mathrm{N})$ will increase by $\sqrt{2}$ if we average $A B$ and $\mathrm{AD}$. If the signal is in $\mathrm{B}$, the signal will be lowered by a factor of two in the $A B+A D$ average. A signal in the $B$ position would also be seen in the BC average with $\sqrt{2}$ increase of the $\mathrm{S} / \mathrm{N}$ in the $\mathrm{BA}+\mathrm{BC}$ average.

In Table 5 we note that the noise level is considerably lower than in the 2004 observations. 
C. M. Persson et al.: The first spectral line surveys searching for signals from the dark ages

Table 5. Upper limits in $\mathrm{mK}$ as a function of angular size on the sky from the spectral surveys observations during 2006/07 (deep searches are not included) using 1, 4 and $16 \mathrm{MHz}$ channel spacings.

\begin{tabular}{|c|c|c|c|c|c|c|c|c|}
\hline \multirow[b]{2}{*}{ Position } & \multicolumn{7}{|c|}{$1 \mathrm{MHz}$ channel spacing } & \multirow[b]{2}{*}{$\begin{array}{c}\text { Peak intensity } \\
{[\sigma]}\end{array}$} \\
\hline & $\begin{array}{c}2-5^{\prime} \\
1 \sigma[\mathrm{mK}]\end{array}$ & $\begin{array}{c}\text { Peak intensity } \\
{[\sigma]}\end{array}$ & $\begin{array}{c}5-10^{\prime} \\
1 \sigma[\mathrm{mK}]\end{array}$ & $\begin{array}{c}\text { Peak intensity } \\
\qquad \sigma]\end{array}$ & $\begin{array}{c}10-15^{\prime} \\
1 \sigma[\mathrm{mK}]\end{array}$ & $\begin{array}{c}\text { Peak intensity } \\
{[\sigma]}\end{array}$ & $\begin{array}{c}15-30^{\prime} \\
1 \sigma[\mathrm{mK}]\end{array}$ & \\
\hline $\mathrm{A}$ & $16(23)$ & $3.0(3.3)$ & $21(31)$ & $3.2(3.4)$ & $21(31)$ & $3.2(3.4)$ & $21(31)$ & $3.2(3.4)$ \\
\hline B & $17(26)$ & $3.1(2.9)$ & $23(34)$ & $3.3(3.2)$ & $23(34)$ & $3.3(3.2)$ & - & - \\
\hline $\mathrm{C}$ & $19(26)$ & $3.3(3.0)$ & $19(26)$ & $3.3(3.0)$ & $25(35)$ & $3.1(3.2)$ & - & - \\
\hline $\mathrm{D}$ & $15(25)$ & $3.1(2.9)$ & $15(25)$ & $3.1(2.9)$ & $20(33)$ & $3.2(3.1)$ & $20(33)$ & $3.2(3.1)$ \\
\hline \multicolumn{9}{|c|}{$4 \mathrm{MHz}$ channel spacing } \\
\hline A & $10(15)$ & $3.0(2.8)$ & $13(20)$ & $3.0(2.9)$ & $13(20)$ & $3.0(2.9)$ & $13(20)$ & $3.0(2.9)$ \\
\hline B & $11(17)$ & $2.7(3.0)$ & $15(23)$ & $2.9(3.1)$ & $15(23)$ & $2.9(3.1)$ & - & - \\
\hline $\mathrm{C}$ & $12(16)$ & $3.0(2.9)$ & $12(16)$ & $3.0(2.9)$ & $16(22)$ & $2.9(3.0)$ & - & - \\
\hline $\mathrm{D}$ & $10(15)$ & $2.9(3.1)$ & $10(15)$ & $2.9(3.1)$ & $13(20)$ & $3.1(3.0)$ & $13(20)$ & $3.1(3.0)$ \\
\hline \multicolumn{9}{|c|}{$16 \mathrm{MHz}$ channel spacing } \\
\hline A & $5(8)$ & $2.7(2.8)$ & $6(11)$ & $3.0(2.9)$ & $6(11)$ & $3.0(2.9)$ & $6(11)$ & $3.0(2.9)$ \\
\hline B & $6(9)$ & $2.8(3.0)$ & $8(12)$ & $2.7(3.1)$ & $8(12)$ & $2.7(3.1)$ & - & - \\
\hline $\mathrm{C}$ & $6(11)$ & $2.7(2.8)$ & $6(11)$ & $2.7(2.8)$ & $8(14)$ & $3.0(2.9)$ & - & - \\
\hline $\mathrm{D}$ & $5(8)$ & $2.9(3.0)$ & $5(8)$ & $2.9(3.0)$ & $7(11)$ & $2.8(3.1)$ & $7(11)$ & $2.8(3.1)$ \\
\hline
\end{tabular}

Notes. The limits are for the data taken with the AOS(AC) and for frequency ranges 542.0-547.5 (486.5-492) GHz.

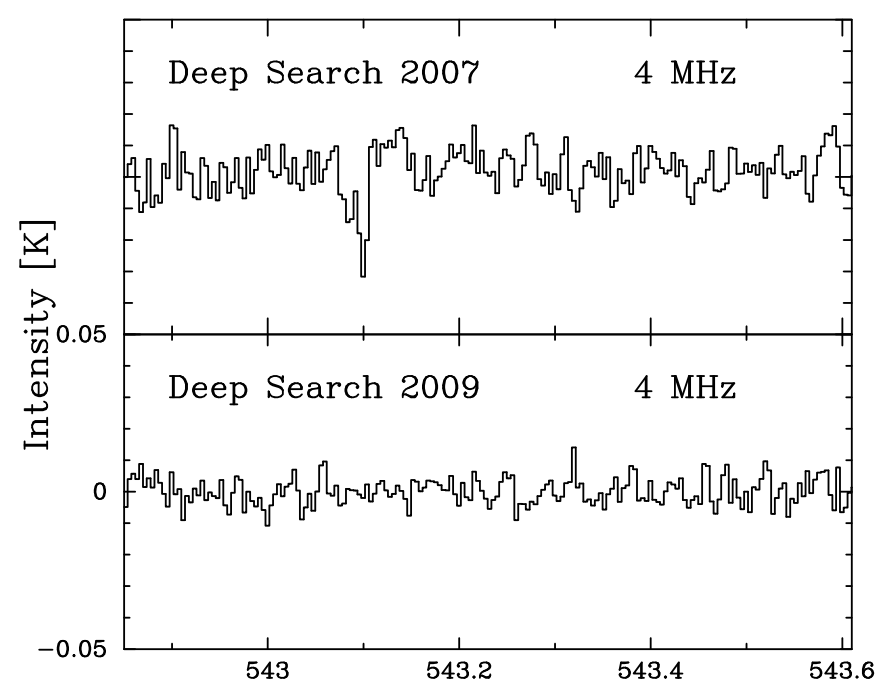

Frequency $[\mathrm{GHz}]$

Fig. 3. Deep searches from 2007 and 2009 observations towards the Hot Spot 1 (position A with position B used as reference) is shown with a channel spacing of $4 \mathrm{MHz}$ with $1 \sigma$ noise levels of 6.5 and $4.0 \mathrm{mK}$, respectively.

\subsection{Comments on results of the deep searches}

The noise level in both deep searches are considerably lower than the 2004 observations and also lower compared to the surveys in 2006/07. The deep search in 2007 resulted in a noise level of $10 \mathrm{mK}$ with $1 \mathrm{MHz}$ channel spacing. Rebinning to 4 and $16 \mathrm{MHz}$ lowers the noise to 6.5 , and $3.5 \mathrm{mK}$, respectively. The noise level in the 2009 deep search produced an even lower noise level of $6.7 \mathrm{mK}$ with $1 \mathrm{MHz}$ channel spacing, and 4.0, and $2.1 \mathrm{mK}$ when rebinning to 4 and $16 \mathrm{MHz}$, respectively.

The possible detection in the 2007 deep search did unfortunately not show up again in the 2009 deep search as seen in Fig. 3. The origin of this feature is difficult to explain but may arise from unknown technical issues with the satellite or the receivers.

\section{Analysis}

In order to interpret our observations in a cosmological context we begin with a summary of the cosmological background and the tools by which we estimate the amplitude of absorption and resonant lines in Sect. 5 as well as their line widths. This is model dependent and we adopt the currently favoured hierarchical $\Lambda \mathrm{CDM}$ cosmology with a cosmological constant in which cold dark matter dominates the evolution of structure. The latest results from five-years of WMAP data (Komatsu et al. 2009) are used, with a Hubble constant $H_{0}=70 \mathrm{~km} \mathrm{~s}^{-1} \mathrm{Mpc}^{-1}$, a dimensionless matter density parameter $\Omega_{\mathrm{M}}=0.274$, a dark energy density parameter $\Omega_{\Lambda}=0.726$, and a baryon density $\Omega_{\mathrm{b}}=0.0456$. This implies a flat geometry where $\Omega_{\mathrm{M}}+\Omega_{\Lambda}=1$.

The proper length $L(z)$ of a primordial perturbation can be estimated by using the small-angle limit of the relation between the angular size $\theta$ that a cloud subtends on the sky and the angular-diameter distance $D_{\mathrm{A}}(z)$ :

$\theta=\frac{L(z)}{D_{\mathrm{A}}(z)} \quad[\mathrm{rad}]$

In a flat Universe, this distance is described by

$D_{\mathrm{A}}(z)=\frac{c}{\left(1+z^{\prime}\right) H_{0}} \int_{0}^{z^{\prime}} \frac{\mathrm{d} z}{\sqrt{\Omega_{\mathrm{M}}(1+z)^{3}+\Omega_{\Lambda}}} \quad[\mathrm{Mpc}]$

where $c$ is the speed of light. Assuming a spherical geometry, the proper length $L(z)$ can be used to calculate the mass of a density perturbation $\Delta \rho / \rho$ following the expansion of the Universe

$M=\frac{4 \pi}{3} \frac{L(z)^{3}}{8} \Omega_{\mathrm{M}} \rho_{\mathrm{cr}}(1+z)^{3}(1+\Delta \rho / \rho)$,

where $\rho_{\text {cr }}=1.88 \times 10^{-29} h^{2} \mathrm{~g} \mathrm{~cm}^{-3}$ is the critical density of the Universe at present time with $h=H_{0} / 100$, and where $\Delta \rho / \rho$ is initially very small but increases with decreasing redshift. Equations $(5)-(7)$ then give a relation between angular size and mass.

The standard structure formation model predicts the formation of gravitationally bound systems from tiny density perturbations via gravitational collapse. The hierarchical model predicts that the smaller perturbations formed first and then 
merged or accreted gas to form even more massive objects. The WMAP5 results are consistent with an epoch of reionisation at $z_{\text {rei }}=10.9 \pm 1.4$ (Komatsu et al. 2009). Later reionisation epochs at $z \sim 6$, are suggested by other kinds of experiments (e.g. Fan et al. 2006). This implies that the first stars must have formed at $z \gtrsim 6$. They are predicted to form in over-dense dark matter regions of $10^{5}-10^{6} M_{\odot}$ at redshifts of about 30-40 (e.g. Glover 2005). These mini-halos may provide a significant, if not dominant, contribution to reionisation at lower redshifts (Choudhury et al. 2008). Mass perturbations $>10^{15} M_{\odot}$ are predicted not yet to have reached their collapse phase.

In the linear regime, the density contrast $\Delta \rho / \rho$ is predicted to grow linearly with the scale factor as $1 /(1+z)$. Sufficiently dense perturbations reach a turn-around point at which their gravity counterbalances the expansion. Then they enter a collapse phase during which the density and temperature quickly increase.

Note that during the linear phase, the only predicted possible signal is by the resonant scattering process, while emission and absorption lines will arise in collapsing perturbations or with high temperature regions for example from the first supernovae and their subsequent hot HII regions as background radiation. The amplitude and line width of all lines depend among many variables on the dynamics of the primordial clouds, which conveniently can be divided into the above mentioned phases: the linear phase, the turn-around phase and the collapse phase.

\subsection{Linear phase}

In the linear phase, the line width will depend on the proper length $L(z)$ of the object. This size will occupy a redshift interval $\Delta z$ in the Hubble flow, and, assuming a spherical geometry and that every part of the cloud moves with the same peculiar velocity, we have (e.g. Maoli et al. 1996; Dubrovich et al. 2008)

$\frac{\Delta v}{v}=\frac{\Delta z}{1+z}=\frac{H_{0} L(z)}{c} \sqrt{\Omega_{\mathrm{M}}(1+z)^{3}+\Omega_{\Lambda}}$,

where $\Delta v$ is the Doppler line width. The line width is thus dependent on the size of the perturbation.

A relationship between line width and mass in the linear phase can now be found using Eq. (7) with $\Delta \rho / \rho \ll 1$ and Eq. (8). The line widths increase with redshift and mass and are very broad, $\Delta v / v \sim 10^{-1}-10^{-5}$, implying line widths of the order of a few thousand $\mathrm{km} \mathrm{s}^{-1}$ (Table C.3 on-line material). Note, that this is only true assuming that the density contrast is very small. When the perturbations grow the line widths will start to deviate from Eq. (8) and become increasingly more narrow. Another addition of uncertainty to the line width is our homogeneity assumption of no substructure within the cloud. Within each mass at its turn-around, there will be smaller mass perturbations which already have reached their respective turn-around. The signals from these regions will be very narrow and superposed on the broader lines from the non-collapsed regions. As a first order approximation in our analysis, we will therefore use a line width of $500 \mathrm{~km} \mathrm{~s}^{-1}$ in our predictions of $\mathrm{HeH}^{+}$resonant lines in the linear phase in Sect. 5.

The intensity of the resonant lines is estimated to be (e.g. de Bernardis et al. 1993; Maoli et al. 1996; Dubrovich et al. 2008)

$$
\frac{\Delta I}{I_{\mathrm{CMB}}}=\left(1-\mathrm{e}^{-\tau}\right)\left(3-\alpha_{v}\right) \frac{v_{\mathrm{p}}}{c} \cos \theta
$$

where $\Delta I$ is the observed intensity, $I_{\mathrm{CMB}}$ the CMB intensity, $\alpha_{v}$ is the spectral index, $v_{\mathrm{p}}$ is the peculiar velocity of the perturbation, $\cos \theta$ is the cosine of the angle with respect to the line of sight of the peculiar velocity, and $\tau$ is the opacity of the transition as calculated in the rest frame of the cloud. The determining factor resulting in a positive or negative sign of $\Delta I / I$ are the $\cos \theta$ factor. The only way to produce resonant scattering "emission" lines is therefore to have a source that moves along the line of sight towards us, while resonant scattering "absorption" lines will appear when the source moves away from us. Note, that the resonant lines do not require temperature differences, but only a moving cloud, $\mathrm{CMB}$ photons and the scattering species.

The spectral index of the CMB $\alpha_{v}=(v / I)(\mathrm{d} I / \mathrm{d} v)$ is derived in Maoli et al. (1996) where $I_{v}=B_{v}\left(T_{\mathrm{CMB}}\right)$ and has the general expression

$\alpha_{v}=3-\frac{h v / k T}{1-\mathrm{e}^{-h v / k T}}$

The peculiar velocity describes the motion of primordial perturbations with respect to the Hubble flow. In the $\Lambda \mathrm{CDM}$ model these perturbations evolve due to potential gradients and increase with time as (Longair 2008)

$v_{\mathrm{p}}=\frac{v_{\mathrm{p} 0}}{\sqrt{1+z}}$

The most commonly used peculiar velocity at present time for the size of a galaxy cluster is $v_{\mathrm{p} 0} \sim 600 \mathrm{~km} \mathrm{~s}^{-1}$, derived from the CMB dipole anisotropy, which together with our spatially flat $\Lambda \mathrm{CDM}$ model, is considered to reproduce the characteristics of the large-scale matter distribution at low redshifts (e.g. Jenkins et al. 1998). Smaller mass perturbations most likely move with a factor of 2-5 higher peculiar velocity (Dubrovich et al. 2008). The present ratio of the peculiar velocity to the speed of light is $\sim 2 \times 10^{-3}$ and decreases with increasing redshift. This is a very limiting factor in the search for resonant lines.

The scattering efficiency of resonant lines is very frequency dependent and depends mainly on two parameters: the number density of the species and the spontaneous transition probability described by the Einstein coefficient $A_{u l}$, where the subscript $u l$ refers to the upper and lower levels of the scattering species. Those species which have a high product of these quantities are of special interest.

The density of the species depends on redshift and can be described in terms of the total density of hydrogen and the fractional abundance of species $x$ as $X_{x}=n_{x} / n_{\mathrm{H}}$. For simplicity, as a first approximation we assume that the density is high enough to allow the number density of the species in an excited state to be described by the Boltzmann distribution

$n_{x, \mathrm{u}}(z)=\frac{g_{\mathrm{u}}}{Q(T)} \mathrm{e}^{-E_{\mathrm{u}} / k T_{\text {ex }}} n_{x}$

where $g_{\mathrm{u}}$ and $E_{\mathrm{u}}$ are the statistical weight and energy of the upper state, respectively, $Q(T)$ is the partition function, $T_{\mathrm{ex}}$ is the excitation temperature, $n_{x}=n_{\mathrm{H}, 0} X_{x, 0}(1+z)^{3}$ with $X_{x, 0}$ as the abundance extrapolated at the present time and the present density of hydrogen atoms $n_{\mathrm{H}, 0}=\rho_{\text {crit }} \Omega_{\mathrm{B}} / m_{\mathrm{p}} \approx 2 \times 10^{-7} \mathrm{~cm}^{-3}$. We thus assume that the molecular abundances have reached their asymptotic limit at the redshifts of interest $\$ 100-200$ on the large scales encompassed by the Odin beam. The possibility of a detection prior to a redshift of a maximum abundance is very limited due to the very low abundances and weak signals at even higher redshifts. On scales smaller than the Odin beam the abundances will certainly vary in regions with higher than average density or by the effects of the first stars. In reality, the population distribution also will depend on the molecular formation 
process, and the radiation field with possible population inversion (maser effects).

The optical depth at the centre of the line can be calculated, assuming LTE and a Gaussian line profile, using

$\tau_{\max }=\int_{l_{1}}^{l_{2}} \sqrt{\frac{\ln 2}{16 \pi^{3}}} \frac{c^{3} n_{x}}{v_{\mathrm{u} l}^{3} \Delta v} \frac{A_{\mathrm{u} l} g_{\mathrm{u}}}{Q(T)} \mathrm{e}^{-E_{\mathrm{u}} / k T_{\mathrm{ex}}}\left(\mathrm{e}^{h v_{\mathrm{u}} / k T_{\mathrm{ex}}}-1\right) \mathrm{d} l,($

where we have taken the stimulated emission into account, and $v_{\mathrm{u} l}$ is the frequency of the transition (cf. Persson et al. 2007). As customary we have converted the line width in frequency $\Delta v$ to a Doppler velocity width $\Delta v$. The integration is performed over the path length of the cloud, $L(z)=l_{2}-l_{1}$. Note, that the path length determines the line width in the strictly linear phase (cf. Table C.3, on-line material).

\subsection{Turn-around phase}

During this phase the tendency of a perturbation to collapse under its gravity just balances its tendency to expand with the rest of the Universe. The perturbation will appear non-moving and all species from every part of the perturbation will emit from the same redshift. This will produce the strongest and the most narrow resonant lines with a line width determined by the thermal broadening (Maoli et al. 1996)

$\frac{\Delta v}{v}=\frac{2}{c} \sqrt{\frac{2 \ln 2 k T_{\mathrm{K}}}{m}}=7.16 \times 10^{-7} \sqrt{\frac{T_{\mathrm{K}}}{A}}$,

where $m$ is the atomic mass and $A$ is the mass in atomic mass units of the species. At low temperatures this implies a line width of a few $\mathrm{km} \mathrm{s}^{-1}$ at high redshifts for $\mathrm{HeH}^{+}$(cf. Table C.3, on-line material).

In addition to this, turbulence will also contribute with an unknown, and perhaps dominating, amplitude. A large region will also have a number of clumps which have narrow lines at slightly different velocities. The total line will be a superposition of these lines which will broaden the line. Finally, the cloud also consists of a number of smaller clouds that already have reached their turn-around which also may act as broadening agents. As a first order approximation in our analysis, we will therefore use a line width of $30 \mathrm{~km} \mathrm{~s}^{-1}$ in our predictions of $\mathrm{HeH}^{+}$resonant lines in the turn-around phase in Sect. 5.

The integrated intensity of the resonant lines has not changed compared to the linear phase, and thus the amplitude, described by Eq. (9), will increase proportionally to the decrease in line width, which could be up to three orders of magnitude.

As pointed out by Dubrovich (1997), a luminescence effect may also produce lines when high energy photons are scattered and decay into several lower energy photons. The amplitude of lines produced by luminescence is given by Eq. (9) times an additional gain coefficient factor $K$. For simple elastic scattering $K=1$ and for luminescence $K \lesssim 1000$. Dubrovich \& Lipovka (1995) investigated luminescence produced by excited rotational-vibrational (ro-vib) transitions of $\mathrm{H}_{2} \mathrm{D}^{+}$which decay to the lowest vibrational state emitting via several rotational transitions. This effect is, however, limited to high temperature or high density regions.

The turn-around and the beginning of the collapse phase are identified as the most favourable for observations of resonant lines (Maoli et al. 1996). It is therefore important to try to estimate at what redshift this will take place (full derivation in Appendix B, on-line material). We assume that the

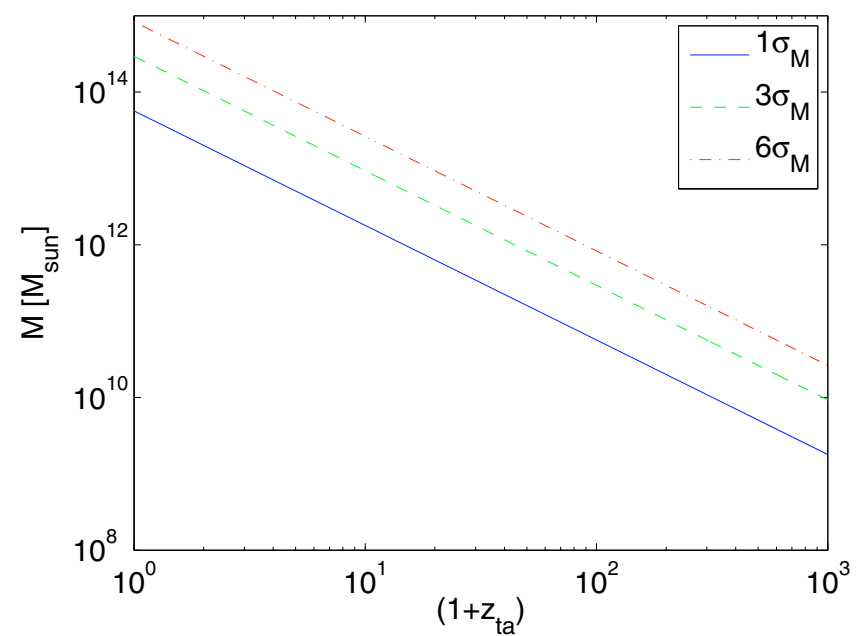

Fig. 4. Turn-around redshift for one, three and six $\sigma_{M}$ mass over-dense primordial perturbations as determined by Eq. (17). Note, that this describes the total mass, and the baryonic to dark matter content is $1 / 6$. Star and galaxy formation starts at later times since the baryonic matter does not collapse immediately at the turn-around redshift.

power spectrum of the dark matter density fluctuations is of Harrison-Zeldovich type, which means

$\sigma_{M}=\left\langle\left(\frac{\Delta M}{M}\right)^{2}\right\rangle^{1 / 2} \sim M^{-2 / 3}$

and that it can be normalised by the observed fluctuations $\sigma_{M_{S}}=10^{-4}$ of the mass $M_{S}=3.72 \times 10^{15} M_{\odot}$ (cf. Eq. (15.13) Longair 2008) within the sound horizon at the last scattering surface where $z_{\text {LSS }}=1090$ giving

$\sigma_{M}=10^{-4}\left(\frac{M_{S}}{M}\right)^{2 / 3}$

The turn-around redshift $z_{\mathrm{TA}}$ for a mass $M$ which has $1 \sigma_{M}$ over density, i.e. $\Delta M / M=\sigma_{M}$, can then be estimated according to

$1+z_{\mathrm{TA}}=1.35\left(1+z_{\mathrm{LSS}}\right)\left(\frac{10^{9} M_{\odot}}{M}\right)^{2 / 3}$.

Figure 4 shows the turn-around redshift $z$ as a function of mass for one, three, and six $\sigma_{M}$ mass perturbations. Note, that the Harrison-Zeldovich power spectrum has the power index $n=1$, and the latest WMAP results (Komatsu et al. 2009) indicate that $n=0.960_{-0.013}^{+0.014}$. In our calculations we have taken the total mass into account, but the baryonic to dark matter mass ratio is only $1 / 6$. The star forming baryonic matter is predicted to subsequently fall into the dark matter potential wells caused by gravitational collapse. Note, that the collapse does not immediately follow when the object arrives at the turn-around point since the infalling mass has difficulties to loose its energy (e.g. Diemand \& Kuhlen 2008).

To be able to quantify the signals from the proto-objects to observables we need to relate the turn-around mass to a linear size corresponding to an angular size of our beam. At the turnaround $(1+\Delta \rho / \rho)=(3 \pi / 4)^{2} \sim 5.55$ and this should be inserted in Eq. (7) to find a mass-linear size relationship at the turnaround. We then use Eqs. (5)-(7) and (17) to find the relation between angular size vs. mass perturbations at turn-around redshift, shown in Fig. 5. 


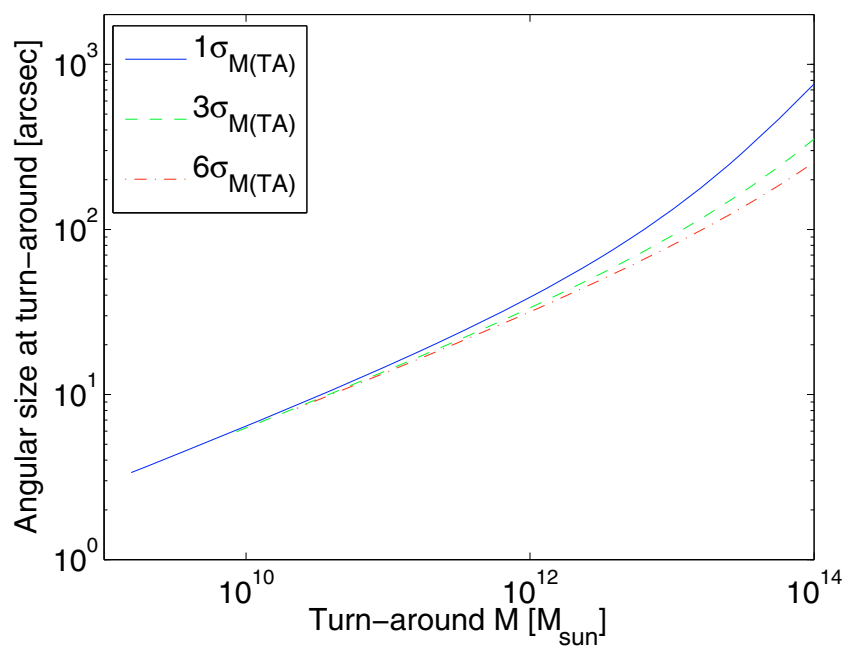

Fig. 5. The angular size vs. total mass at $z_{\mathrm{TA}}$, assuming one, three and six $\sigma_{M}$ over densities. Note, that this describes the total mass.

\subsection{Non-linear collapse phase}

When $(1+\Delta \rho / \rho) \gtrsim(3 \pi / 4)^{2}$ the collapse will begin. The amplitude and line width now depend on the collapse rate in addition to the peculiar velocity. Depending on the ratio of the two velocities resonant lines can appear in absorption, emission or have a double peak (a summary is found in Maoli et al. 1996). During the initial stages the line widths are expected to increase compared to the narrow width during the turn-around. This is also the only evolutionary stage where the peculiar velocity is no longer required to produce resonant lines.

The chemical abundances could also be substantially modified during the collapse phase of a primordial cloud when the increasing temperature and density induce new chemistry. In such regions, molecules will both be destroyed and produced (Puy \& Signore 1996). The first Pop III objects will quickly create a complex, multi-phase interstellar medium with a large range of densities and temperatures; up to 6 orders of magnitude at a given radius (Wise \& Abel 2008).

The very energetic radiation from the first collapsed objects can for instance dissociate molecular hydrogen (e.g. Haiman et al. 1997, 2000). However, if the amount of $\mathrm{H}_{2}$ produced in the cooling gas behind shock waves from the first supernova explosions exceeds the destroyed primordial $\mathrm{H}_{2}$ inside the photodissociation regions, the first objects would have a net positive feedback on molecular production and galaxy formation. Ferrara (1998) concludes that multi-supernova explosions propagating through the interstellar medium can produce regions with a very high $\mathrm{H}_{2}$ abundance. For a wide range of physical parameters in such regions he found an $\mathrm{H}_{2}$ fraction of about $6 \times 10^{-3}$. In addition, extremely energetic pair-instability supernovae $\left(140-260 M_{\odot}\right)$ produced large amounts of dust at very early times (e.g. Schneider et al. 2004) thereby allowing an increased molecular production including $\mathrm{H}_{2}$ and $\mathrm{CO}$. All these effects influence the amplitudes of the primordial resonant lines and thermal emission and absorption. There are, however, large uncertainties in the complex $\mathrm{H}_{2}$ and HD chemistry (Glover \& Abel 2008).

The resonant scattering will become suppressed when the density becomes higher than some critical density depending on species, since frequent collisions will cause thermal radiation to dominate over resonant scattering (Basu 2007). The increasing numbers of collisions will drive the level populations and the excitation towards the lower (or higher) matter temperature, and should enhance the spectral line absorption (or emission). This effect will be greatest in molecules like $\mathrm{H}_{2}$ and $\mathrm{HD}$, which have zero or small dipole moments and thus weak transitions.

\section{Sensitivity analysis}

Our observations have given us upper limits in terms of noise levels, which we now want to analyse using Eq. (9). This equation is, however, expressed in intensity while sub-mm and radio antennas are usually calibrated according to the brightness temperature in the Rayleigh-Jeans limit in terms of (Rohlfs \& Wilson 2004)

$T_{\mathrm{b}}=\frac{c^{2}}{2 k v^{2}} I_{v}=\frac{\lambda^{2}}{2 k} I_{v}$

Thus, the solution of the radiative transport equation with a constant source function, in our switched observations between signal and reference positions, becomes (cf. Persson et al. 2009)

$$
\Delta T_{\mathrm{A}}^{*}=T_{\mathrm{b}} \eta_{\mathrm{mb}} \eta_{\mathrm{bf}}=\left[J\left(T_{\mathrm{ex}}\right)-J\left(T_{\mathrm{bg}}\right)\right]\left(1-\mathrm{e}^{-\tau}\right) \eta_{\mathrm{mb}} \eta_{\mathrm{bf}},
$$

where $\Delta T_{\mathrm{A}}^{*}$ is the observed antenna temperature, $\eta_{\mathrm{mb}}$ is the main beam efficiency, $\eta_{\text {bf }}$ describes the beam filling (see Eq. (21) below), $T_{\text {ex }}$ and $T_{\mathrm{bg}}$ are the excitation and background temperatures, and the radiation temperature, $J(T)$ is (Rohlfs \& Wilson 2004)

$$
J(T)=\frac{h v}{k} \frac{1}{\mathrm{e}^{h v / k T}-1} \sim T .
$$

Note that the approximation is only valid if $h v \ll k T$ which is often used at frequencies $\$ 100 \mathrm{GHz}$ and temperatures much higher than the CMB. Figure A.3 (on-line material) shows $J\left(T_{\mathrm{CMB}}\right)$ as a function of frequency with $T=2.725 \mathrm{~K}$. Around $550 \mathrm{GHz}$, the approximation is no longer valid due to the low (CMB) temperature and high frequencies which lowers $J\left(T_{\mathrm{CMB}}\right)$ to $\sim 10^{-3}$. Accordingly, we use $J\left(T_{\text {ex }}\right)$ and not $T_{\text {ex }}$ in our calculations. The $5 \sigma$ noise level from Tables 3 and 5 is used as $\Delta T_{\mathrm{A}}^{*}$ analysing the observations.

The beam filling $\eta_{\text {bf }}$ can be estimated by (cf. Persson et al. 2009)

$\eta_{\mathrm{bf}}=\frac{\theta_{\mathrm{s}}^{2}}{\theta_{\mathrm{s}}^{2}+\theta_{\text {beam }}^{2}}$,

where $\theta_{\mathrm{s}}$ is the $F W H M$ angular size of the circular source, and $\theta_{\text {beam }}$ is the $F W H M$ beam size. If the source is much smaller than the beam we will severely suffer from beam dilution by this factor. We therefore assume that the primordial perturbation fills the large Odin beam $\left(\eta_{\mathrm{bf}}=1\right)$ thereby determining the minimum size of a perturbation to which we are sensitive (Figs. 4 and 5). In Table C.2 (on-line material) the physical sizes corresponding to the Odin beam as well as the step sizes in the 2006/07 observations are given for a number of redshifts. According to Figs. 4 and 5, the Odin beam size is most sensitive to about $6 \times 10^{12}-10^{13} M_{\odot}$ perturbations with turn-around redshifts of about $3-10(1-3 \sigma)$.

\subsection{Resonant lines}

Equation (9) gives the intensity of the resonant lines. To express this in Rayleigh-Jeans (RJ) brightness temperature we use Eq. (19) and obtain

$T_{\mathrm{b}}=J\left(T_{\mathrm{CMB}}\right)\left(1-\mathrm{e}^{-\tau}\right)\left(3-\alpha_{v}\right) \frac{v_{\mathrm{p}}}{c} \cos \theta$. 
Table 6. Estimated largest possible brightness temperature $T_{\mathrm{b}}$ for optically thick resonant lines, and $v_{p}(z)=600 / \sqrt{1+z} \mathrm{~km} \mathrm{~s}^{-1}$.

\begin{tabular}{cccc}
\hline \hline Obs. Freq. & \multicolumn{3}{c}{ Maximum $\left|T_{\mathrm{b}}\right|[\mathrm{mK}]$} \\
{$[\mathrm{GHz}]$} & $z=10$ & $z=30$ & $z=100$ \\
\hline 1 & 1.6 & 0.98 & 0.54 \\
50 & 1.5 & 0.92 & 0.51 \\
100 & 1.3 & 0.76 & 0.42 \\
200 & 0.64 & 0.38 & 0.21 \\
300 & 0.24 & 0.14 & 0.078 \\
400 & 0.071 & 0.042 & 0.024 \\
500 & 0.019 & 0.011 & 0.006 \\
600 & 0.005 & 0.003 & 0.002 \\
\hline
\end{tabular}

Notes. This velocity is typical for clusters while for a smaller mass it could be higher. Note, that these values are the same for all species and transitions.

Note, that the observed antenna temperature is lower by a factor $\eta_{\mathrm{bf}} \eta_{\mathrm{mb}}$ (Eq. (19)).

To estimate the maximum RJ brightness temperature possible we assume that the lines in Eq. (22) are optically thick $(\tau>1)$ since this is a limit after which the line will no longer increase its amplitude. The results are given in Table 6 with an accuracy depending on the uncertainty of the assumed peculiar velocities. Obviously, even taking this uncertainty into account, the resonant lines would be extremely difficult to detect at frequencies around $500 \mathrm{GHz}$ and require noise levels orders of magnitudes lower than our observations. The Odin main beam temperature $T_{\mathrm{mb}}$-scale is related to the flux density by $F_{v} / T_{\mathrm{mb}}=2600 \mathrm{Jy} / \mathrm{K}$ at $543 \mathrm{GHz}$. Figure A.3 (on-line material) shows $J\left(T_{\mathrm{CMB}}\right) \times(3-\alpha)$, and demonstrates that we loose almost two orders of magnitude in sensitivity around the observed Odin frequencies compared to frequencies below $200 \mathrm{GHz}$. This is a very limiting factor for detecting the already weak resonant lines, especially for the rotational-vibrational transitions which cannot be observed below $200-400 \mathrm{GHz}$ if they form around $z=200-400$. The conclusion is therefore that the rotational-vibrational transitions of resonant lines most likely will be too weak for a detection even with future much more sensitive antennas.

At frequencies $\$ 100-200 \mathrm{GHz}$ the amplitudes of the resonant lines are orders of magnitudes higher than around $500-600 \mathrm{GHz}$, and hence a detection may be possible if the opacity is high at low frequencies. However, the resonant lines suffer from their dependence on the peculiar velocity which lowers their amplitudes by approximately three orders of magnitude at $z=0$, and even more at higher redshifts (Eqs. (11) and (22)). Since thermal emission or absorption lines do not depend on peculiar velocities, if existing, they have the potential to be much stronger than the resonant lines.

Note, that these results also need to be corrected for beam filling, if the object is smaller than the beam, and for the beam efficiency to obtain the observable antenna temperature. For the Odin satellite this only lowers the amplitude by $10 \%$, but for other antennas the reduction may be more than $50 \%$.

We also estimate the amplitude of optically thin resonant lines. As an example, with the main goal to show the pattern of the resonant lines as a function of redshift and to get a first rough estimate of the amplitudes, we consider the rotational and first branch of rotational-vibrational resonant $\mathrm{HeH}^{+}$lines for four different redshifts, shown in Fig. 6. A single perturbation is assumed, with a size filling the $2: 1$ Odin beam. We have assumed a low density of hydrogen equal to the present one: $n_{\mathrm{H}}=2 \times 10^{-7}(1+z)^{3}$ at redshifts $\geq 30$, and increased this density 5.55 times at $z=10$ (density at turnaround). We have used an $\mathrm{HeH}^{+}$abundance relative to hydrogen of $X\left(\mathrm{HeH}^{+}\right)=10^{-12}$ and the background temperature follows the $\mathrm{CMB}$ at all redshifts. The adopted lines widths are narrow with $\Delta v=30 \mathrm{~km} \mathrm{~s}^{-1}$ at $z=10$, and broad with $\Delta v=500 \mathrm{~km} \mathrm{~s}^{-1}$ from $z \geq 30$ as indicated in the legends. This choice of densities and line widths arises from the fact that for $z \gtrsim 10$, the large Odin beam corresponds to much larger physical sizes and masses than the turn-around objects (Fig. 4), and at higher redshifts we are therefore most sensitive to objects in their linear phase. The peculiar velocity is described by Eq. (11) with $v_{\mathrm{p} 0} \sim 600 \mathrm{~km} \mathrm{~s}^{-1}$.

Since the CMB temperature follows $2.725(1+z) \mathrm{K}$, this implies that high redshift sources have an increased ability to populate higher molecular energy levels since the rising temperatures give the photons more energy to excite the molecules. This is shown in Fig. 6 where high molecular transitions are increasingly populated towards higher redshifts. The rotationalvibrational transitions are not seen at all until $z \sim 300$, and are always much weaker than the rotational lines at all redshifts. It is also clearly seen that lines observed at frequencies around $500 \mathrm{GHz}$ always will be weaker than around $100 \mathrm{GHz}$; a clear disadvantage for our current observations. This could to some extent be compensated by the previously mentioned luminescence process (Dubrovich 1997) but has not been considered here.

We should here stress that the predictions of Fig. 6 are based upon rather conservative assumptions. Any increase of abundance and density, or decrease of line width, would directly increase the amplitudes of the $\mathrm{HeH}^{+}$signals. Note also that the abundance is a function of redshift and that we here simply have assumed that the abundance has reached a maximum value. The $\mathrm{HeH}^{+}$abundance is indeed very uncertain with a predicted abundance range of $10^{-8}-10^{-15}$ (Dubrovich et al. 2008; Vonlanthen et al. 2009).

\subsection{Thermal absorption lines}

The advantages of the Odin satellite are the absence of adverse effects from the terrestrial atmosphere and the tunable SSB receivers, allowing a spectral coverage of a broad band. However, one severe disadvantage for detection of resonant lines with Odin is the high frequencies. To detect resonant scattering lines the best observing frequencies are below 100-200 GHz considering the rapid decrease of the radiation temperature as shown in Fig. A.3 (on-line material).

There are, however, other possibilities of detecting signals from the dark ages. Thermal emission or absorption depend on the competition between radiation and collisions as given by the radiative transfer equation

$T_{\mathrm{b}}=\frac{h v_{\mathrm{obs}}}{k}\left(\frac{1}{\mathrm{e}^{h v_{\mathrm{u} l} / k T_{\mathrm{ex}}}-1}-\frac{1}{\mathrm{e}^{h v_{\mathrm{ul}} / k T_{\mathrm{bg}}}-1}\right)\left(1-\mathrm{e}^{-\tau}\right)$,

where $T_{\mathrm{b}}$ is the Rayleigh-Jeans brightness temperature, $T_{\mathrm{ex}}$ is the excitation temperature of a transition and $T_{\mathrm{bg}}$ is the background radiation. Note that the latter two temperatures, as well as the opacity, have to be determined at the high redshift source, and that the frequencies on the right side in Eq. (23) are not the same. The excitation temperature depends on the radiation field, which is the CMB at high redshifts, and at lower redshifts it is dominated by the energetic radiation from the first star formation, and on the densities through collisions. The frequency in the first factor is the redshifted rest frequency of the transition 

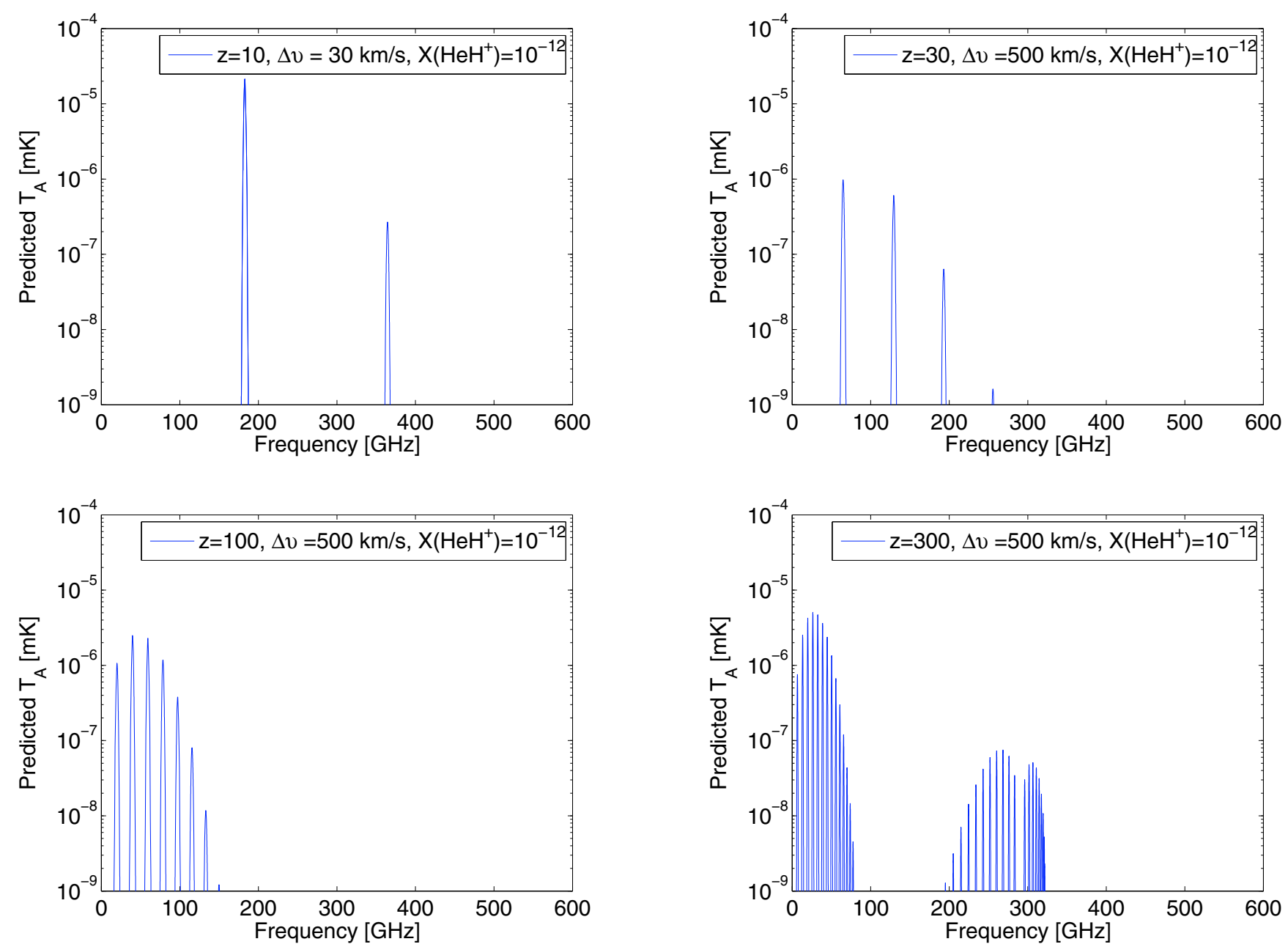

Fig. 6. These plots are an example with the main goal to give an idea of the pattern of the primordial spectral lines: predicted antenna temperature $\Delta T_{\mathrm{A}}^{*}$ of resonant $\mathrm{HeH}^{+}$optically thin lines within the 2.1 Odin beam at $z=10,30,100$, and 300. Turn-around phase is assumed for $z=10$ and for higher $z$ we assume linear phase with line widths as indicated in the legends. We also assume a low density $n_{\mathrm{H}}=2 \times 10^{-7}(1+z)^{3}$ and $X\left(\mathrm{HeH}^{+}\right)=10^{-12}$. Note, that any increase of the density, abundance, or decrease of line width, directly increases the amplitude of the signals with an equal magnitude. If thermal absorption processes are present, they may be several orders of magnitudes stronger than the resonant lines. The amplitudes of the ro-vibrational lines are lower than $10^{-8} \mathrm{mK}$ until $z \sim 300$, when they show up around 200 to $300 \mathrm{GHz}$. They are, however, orders of magnitudes weaker than the rotational lines.

which is the same as the observed frequency, while the other factors include the rest frequency of the transition at the high redshift source.

Equation (23) states that a strong signal requires a large temperature difference between the excitation and background temperatures, which can be produced by for instance collapsing primordial perturbations or the more rapid cooling of the gas temperature compared to the CMB at even higher redshifts. If $T_{\text {ex }} \ll T_{\text {bg }}$ Eq. (23) simplifies to

$T_{\mathrm{b}}=-\frac{h v_{\mathrm{obs}}}{k} \frac{1}{\mathrm{e}^{h v_{\mathrm{ul}} / k T_{\mathrm{bg}}}-1}\left(1-\mathrm{e}^{-\tau}\right)$.

The maximum Rayleigh-Jeans brightness temperature of an absorption line can be estimated using Eq. (24) and assuming an optically thick line, which will cause the last factor to approach unity. The largest possible $T_{\mathrm{b}}$ as a function of observation frequency when the background temperature is the $\mathrm{CMB}$ at the corresponding redshift are given in Table 7. We have also calculated the largest possible intensity when the excitation temperature is only $10 \%$ and $2 \%$ less than the CMB using Eq. (23). Note, that to calculate the observed antenna temperature the brightness temperature needs to be corrected with the (unknown) beamfilling and beam-efficiency using Eq. (19).

Also note that if $T_{\mathrm{bg}}=T_{\mathrm{CMB}}(z)$, the argument of the exponential functions in Eq. (24) can be written as

$\frac{h v_{\mathrm{u} l}}{k T_{\mathrm{CMB}}}=\frac{h v_{\mathrm{u} l}}{k T_{0}(1+z)}=\frac{h v_{\mathrm{obs}}}{k T_{0}}$

where $T_{0}$ is the CMB temperature today. This implies that the maximum strength of an absorption line is redshift independent assuming an optically thick transition. In this case, at a chosen observation frequency the amplitude is the same for all species and transitions and is only determined by the known curve $J\left(T_{0}\right)$ in Fig. A.3 (on-line material).

If the background temperature is not the $\mathrm{CMB}$, the argument of the exponential function then becomes

$\frac{h v_{\mathrm{u} l}}{k T_{\mathrm{bg}}}=\frac{h v_{\mathrm{obs}}(1+z)}{k T_{\mathrm{bg}}}$

As seen, the maximum absorption strength in this case depends on both the redshift and the unknown background temperature. 
Table 7. Estimated largest possible absorption intensity $T_{\mathrm{b}}$ at different observing frequencies for optically thick absorption lines assuming that the background temperature is the CMB.

\begin{tabular}{cccc}
\hline \hline Obs. Freq. & \multicolumn{3}{c}{ Max intensity $\left|T_{\mathrm{b}}\right|$} \\
{$[\mathrm{GHz}]$} & $\begin{array}{c}T_{\mathrm{ex}}=0 \\
{[\mathrm{mK}]}\end{array}$ & $\begin{array}{c}T_{\mathrm{ex}}=0.90 \times T_{\mathrm{CMB}} \\
{[\mathrm{mK}]}\end{array}$ & $\begin{array}{c}T_{\mathrm{ex}}=0.98 \times T_{\mathrm{CMB}} \\
{[\mathrm{mK}]}\end{array}$ \\
\hline 1 & 2700 & 270 & 54 \\
50 & 1700 & 250 & 51 \\
100 & 1000 & 210 & 42 \\
200 & 290 & 97 & 21 \\
300 & 73 & 33 & 7.5 \\
400 & 17 & 9.1 & 2.2 \\
500 & 3.6 & 2.2 & 0.6 \\
600 & 0.74 & 0.5 & 0.1 \\
\hline
\end{tabular}

Notes. We have calculated this using Eq. (23) and $T_{\mathrm{ex}}=0, T_{\mathrm{ex}}=$ $0.90 \times T_{\mathrm{CMB}}$ and $T_{\mathrm{ex}}=0.98 \times T_{\mathrm{CMB}}$. Note that these values are redshift independent.

The noise levels in our observations can be used to set upper limits on possible absorption lines at the observed frequencies. The background source cannot, however, be the CMB at these frequencies since the maximum possible absorption intensity of such a signal is about $3 \mathrm{mK}$ (Table 7). This corresponds to about $1 \sigma$ of our best observations (deep searches), and even less for the spectral line surveys. Thus, the analysis in order to put upper limits using the Odin observations depends on both the unknown background temperature and the redshift, in addition to the opacity and excitation temperature. We give a very simple example of such an analysis in Table 8 . We have here estimated the required background temperature (other than the CMB) in order to produce an absorption line with $T_{\mathrm{b}}=10 \mathrm{mK}$ at an observing frequency of $550 \mathrm{GHz}$ for some molecular transitions. We have used two different opacities and assumed that $T_{\text {ex }} \ll T_{\text {bg }}$ (Eq. (24)). Such an absorption line would correspond to about a $5 \sigma$ detection for our 2009 deep search using $16 \mathrm{MHz}$ channel spacing, or a $1-2 \sigma$ detection for our line surveys using $16 \mathrm{MHz}$ channel spacings (Table 5). We have also listed the CMB temperature at the corresponding redshift as a comparison. A more sophisticated modelling, including excitation temperatures and estimated opacities, is demanded to produce more realistic results and to investigate how the intensities of different transitions vary with the physical conditions at different epochs.

\section{Summary}

In order to constrain cosmological models of star and structure formation as well as the chemical evolution in the early Universe, we have performed spectral line surveys towards several positions without any known sources of emission in a search for primordial spectral lines from the Dark Ages. The first survey covered a broad band of $31 \mathrm{GHz}$ between $547-578 \mathrm{GHz}$ towards two positions with fixed reference-positions. The second survey covered $11 \mathrm{GHz}$ in the bands $542.0-547.5 \mathrm{GHz}$ and 486.5-492.0 GHz towards four positions. We also performed two deep searches towards one position with 543.100 and $543.250 \mathrm{GHz}$ as centre frequencies. No lines were detected, and thus the results are upper limits in terms of noise level. Typical $1 \sigma$ values are $5-35 \mathrm{mK}$ in the $11 \mathrm{GHz}$ survey, 14-90 $\mathrm{mK}$ in the $31 \mathrm{GHz}$ survey, and 2-7 $\mathrm{mK}$ in the deep searches over a $1 \mathrm{GHz}$ band.

The major improvement made by the Odin observations compared to de Bernardis et al. (1993) is the broad bandwidth
Table 8. Estimated required background temperatures to produce absorption lines with $T_{\mathrm{b}}=-10 \mathrm{mK}$ at an observing frequency of $550 \mathrm{GHz}$ assuming $T_{\text {ex }} \ll T_{\mathrm{bg}}$, and an opacity of one or $10^{-3}$.

\begin{tabular}{lccrr}
\hline \hline Transition & Redshift & Opacity & $T_{\mathrm{bg}}$ & $T_{\mathrm{CMB}}$ \\
& $z$ & $\tau$ & {$[\mathrm{K}]$} & {$[\mathrm{K}]$} \\
\hline $\mathrm{H}_{2}(2-0)$ & 18.3 & 1 & 58 & 53 \\
& & $10^{-3}$ & 260 & \\
$\mathrm{H}_{2}(3-1)$ & 31.0 & 1 & 96 & 87 \\
& & $10^{-3}$ & 430 & \\
$\mathrm{H}_{2}(4-2)$ & 43.4 & 1 & 132 & 121 \\
& & $10^{-3}$ & 595 & \\
$\mathrm{H}_{2}(5-3)$ & 55.4 & 1 & 168 & 154 \\
& & $10^{-3}$ & 756 & \\
$\mathrm{HD}(4-3)$ & 18.1 & 1 & 57 & 52 \\
& & $10^{-3}$ & 257 & \\
$\mathrm{HD}(5-4)$ & 22.7 & 1 & 71 & 65 \\
& & $10^{-3}$ & 317 & \\
$\mathrm{HeH}^{+}(4-3)$ & 13.4 & 1 & 43 & 39 \\
& & $10^{-3}$ & 194 & \\
$\mathrm{HeH}^{+}(5-4)$ & 16.7 & 1 & 54 & 48 \\
& & $10^{-3}$ & 240 & \\
\hline
\end{tabular}

Notes. Note, that since the observing frequencies are the same for all transitions the source redshift is different for every transition. As a comparison, the $\mathrm{CMB}$ temperatures are listed for each redshift.

covered allowing a wide range of redshifts to be explored for a number of atomic and molecular species. In addition, in the second survey we have taken into account the unknown sizes of the clouds by testing an observational strategy where we have observed towards four positions in a sequence with different angular distances between the reference and signal position. An important benefit of our observations is that we do no suffer from spectral line contamination from the terrestrial atmosphere.

At low densities and in matter-radiation equilibrium conditions, the only expected signal is from resonant line scattering between $\mathrm{CMB}$ photons and matter moving with respect to the expansion of the Universe. These lines suffer, however, from the dependence of the low CMB temperature and the peculiar velocities of the moving primordial perturbations which is about $10^{-3}$ at present and even lower at higher redshifts. In order to obtain an estimate of the highest possible intensities we assume optically thick resonant lines and no beam-dilution. The intensities will then be at most of the order of a few $\mathrm{mK}$ at frequencies below 100-200 GHz, and orders of magnitude lower at higher frequencies $(5 \mu \mathrm{K}$ at $600 \mathrm{GHz})$. Since the lines most likely have $\tau \ll 1$ this will further lower their intensities with orders of magnitudes.

If existing, thermal absorption lines on the other hand have the potential to be observable at both high and low frequencies. Such lines do not suffer from the dependence of peculiar velocities of the moving primordial perturbations. The background radiation could also be considerably higher than the CMB, thereby producing stronger lines. The background could for instance be collapsing primordial perturbations, the first stars which are predicted to form at $z \sim 20-30$, or the remnants of pop III supernovae. These objects probably emitted large amounts of energetic radiation which foreground primordial perturbations could absorb. Also here we find the highest possible intensities by assuming optically thick lines and no beam-dilution. Assuming that the CMB is the background radiation, we find that the largest possible intensity is $1-2 \mathrm{~K}$ below $100 \mathrm{GHz}$, and of the order of a few $\mathrm{mK}$ around $500 \mathrm{GHz}$. If the background radiation is higher than the CMB, the intensities will increase. Also in this case the 
opacity most likely is $\ll 1$ which will lower the absorption line intensities, in addition to beam-dilution.

The strength and line-width of the primordial lines depend on the evolution of the primordial perturbations. In the first (linear) phase they are very broad and weak, but become increasingly stronger and more narrow during the evolution of the cloud. The turn-around phase, which produces the strongest and most narrow lines, and the beginning of the collapse have previously been identified as the most favourable evolutionary phases for observations (Maoli et al. 1996). We have made a simple estimation of the redshifts and sizes of the primordial perturbations at their turn-around phase, and find that the Odin beam size of 2 '. 1 corresponds to a turn-around mass of about $6 \times 10^{12} M_{\odot}$ at a corresponding redshift of about 3 at a $1 \sigma$ level. Smaller perturbations at higher redshift will thus suffer from beam-dilution. A beam-size of $40^{\prime \prime}$, which is approximately the beam-size of Herschel Space Observatory ${ }^{5}$, corresponds to a $1 \sigma$ turn-around mass and redshift of about $10^{12} M_{\odot}$ and $z \sim 10$.

The lowest rotational transitions of $\mathrm{H}_{2}$ will fall in the Odin band around $500 \mathrm{GHz}$ from $z \sim 20-30$, while the lowest transitions of other species such as $\mathrm{HD}, \mathrm{HD}^{+}$, and $\mathrm{HeH}^{+}$will fall in the band around $100 \mathrm{GHz}$ from the same epoch. These transitions may be searched for using ground based antennas - even though the foreground and atmospheric radiation will pose a problem.

An important aspect of our work has been to test different observational strategies to prepare for our forthcoming observations with the much more sensitive telescope and receivers aboard the Herschel Space Observatory launched on May 14, 2009. The much lower noise level and the even broader band coverage with Herschel will increase the possibility of a detection. Other interesting facilities to consider in the searches for primordial molecules are for example the Atacama Large Millimeter Array ${ }^{6}$ (ALMA), the Combined Array for Research in Millimeter-Wave Astronomy ${ }^{7}$ (CARMA), the IRAM Plateau de Bure Interferometer and the IRAM 30-m telescope ${ }^{8}$, and the Very Large Array $^{9}$ (VLA) at 20-45 GHz. Our observing methods and resulting limits, paired with a sensitivity analysis taking into account the evolution of primordial perturbations, should be a valuable input to the planning of these observations.

Spectral lines from primordial atoms and molecules may very well be the only way to probe the epoch of the cosmic Dark Ages and its end when the first stars formed. The search for these primordial signals is a very difficult task indeed, but a detection could be possible with the use of future facilities and would introduce an additional important way to discriminate between models of the early universe as well as star and structure formation.

\footnotetext{
5 http://herschel.esac.esa.int/

6 http://www . alma.info/

7 http://www . mmarray.org/

8 http://www . iram. fr/

9 http://www.vla.nrao.edu/
}

Acknowledgements. Research on resonant primordial lines started in our collaboration by Francesco Melchiorri nearly twenty years ago. Now that he left us we miss his enthusiasm and creativity and we would like to dedicate this work to his memory. Many thanks also to John H. Black and Per Bergman for valuable comments and discussions, and to the whole Odin team. We also thank the anonymous referee whose constructive comments led to an improvement of the paper. Generous financial support from the Research Councils and Space Agencies in Sweden, Canada, Finland and France is gratefully acknowledged.

\section{References}

Abel, T., Bryan, G. L., \& Norman, M. L. 2000, ApJ, 540, 39 Applegate, J. H., Hogan, C. J., \& Scherrer, R. J. 1987, Phys. Rev. D, 35, 1151 Barkana, R., \& Loeb, A. 2001, Phys. Rep., 349, 125 Basu, K. 2007, New Astron. Rev., 51, 431

Basu, K., Hernández-Monteagudo, C., \& Sunyaev, R. A. 2004, A\&A, 416, 447 Baugh, C. M. 2006, Rep. Prog. Phys., 69, 3101

Becker, R. H., Fan, X., White, R. L., et al. 2001, AJ, 122, 2850

Bertoldi, F., Cox, P., Neri, R., et al. 2003, A\&A, 409, L47

Black, J. H. 2006, in Faraday Discussions of the Royal Society of Chemistry

(UK), Chemistry and cosmology, 133, 27

Bovino, S., Wernli, M., \& Gianturco, F. A. 2009, ApJ, 699, 383

Bowman, J. D., Morales, M. F., \& Hewitt, J. N. 2009, ApJ, 695, 183

Bromm, V., \& Larson, R. B. 2004, ARA\&A, 42, 79

Bromm, V., Yoshida, N., Hernquist, L., \& McKee, C. F. 2009, Nature, 459, 49

Campos, J. C., Saucedo Morales, J. C., Lipovka, A. A., \& Nunes-Lopez, R. 2007,

in IAU Symp. 235, ed. F. Combes, \& J. Palous, 413

Cherchneff, I., \& Lilly, S. 2008, ApJ, 683, L123

Cherchneff, I., \& Dwek, E. 2009, ApJ, 703, 642

Chluba, J., \& Sunyaev, R. A. 2006, A\&A, 458, L29

Chluba, J., Rubiño-Martín, J. A., \& Sunyaev, R. A. 2007, MNRAS, 374, 1310

Choudhury, T. R., Ferrara, A., \& Gallerani, S. 2008, MNRAS, 385, L58

Ciardi, B., \& Ferrara, A. 2005, Space Sci. Rev., 116, 625

de Bernardis, P., Masi, S., Melchiorri, B., \& Melchiorri, F. 1990, ApJ, 357, 8

de Bernardis, P., Dubrovich, V., Encrenaz, P., et al. 1993, A\&A, 269, 1

De Lucia, G., \& Poggianti, B. M. 2008, in ASP Conf. Ser., ed. T. Kodama, T.

Yamada, \& K. Aoki, 399, 314

Diemand, J., \& Kuhlen, M. 2008, ApJ, 680, L25

Dubrovich, V., Bajkova, A., \& Khaikin, V. B. 2008, New Astron., 13, 28

Dubrovich, V. K. 1977, Sov. Astron. Lett., 3, 128

Dubrovich, V. K. 1997, A\&A, 324, 27

Dubrovich, V. K., \& Lipovka, A. A. 1995, A\&A, 296, 301

Dunkley, J., Komatsu, E., Nolta, M. R., et al. 2009, ApJS, 180, 306

Ellis, R., \& Silk, J. 2007 [arXiv:0712 .2865]

Fan, X., Strauss, M. A., Becker, R. H., et al. 2006, AJ, 132, 117

Ferrara, A. 1998, ApJ, 499, L17

Fixsen, D. J., Cheng, E. S., Gales, J. M., et al. 1996, ApJ, 473, 576

Frisk, U., Hagström, M., Ala-Laurinaho, J., et al. 2003, A\&A, 402, L27

Frye, B. L., Bowen, D. V., Hurley, M., et al. 2008, ApJ, 685, L5

Furlanetto, S. R., Lidz, A., Loeb, A., et al. 2009, Astron., 2010, 83

Galli, D., \& Palla, F. 1998, A\&A, 335, 403

Galli, D., \& Palla, F. 2002, Planet. Space Sci., 50, 1197

Glover, S. 2005, Space Sci. Rev., 117, 445

Glover, S. C. O., \& Abel, T. 2008, MNRAS, 388, 1627

Glover, S. C. O., Clark, P. C., Greif, T. H., et al. 2008, in IAU Symp., ed. L. K. Hunt, S. Madden, \& R. Schneider, 255, 3

Gosachinskij, I. V., Dubrovich, V. K., Zhelenkov, S. R., Il'in, G. N., \& Prozorov, V. A. 2002, Astron. Rep., 46, 543

Greif, T. H., Johnson, J. L., \& Bromm, V. 2008a, in First Stars III, ed. B. W. O'Shea, \& A. Heger, AIP Conf. Ser., 990, 405

Greif, T. H., Johnson, J. L., Klessen, R. S., \& Bromm, V. 2008b, MNRAS, 387, 1021

Haiman, Z., Rees, M. J., \& Loeb, A. 1997, ApJ, 476, 458

Haiman, Z., Abel, T., \& Rees, M. J. 2000, ApJ, 534, 11

Hogan, C. J., \& Rees, M. J. 1979, MNRAS, 188, 791

Jee, M. J., Rosati, P., Ford, H. C., et al. 2009, ApJ, 704, 672

Jenkins, A., Frenk, C. S., Pearce, F. R., et al. 1998, ApJ, 499, 20

Johnson, J. L., \& Bromm, V. 2006, MNRAS, 366, 247

Kamaya, H., \& Silk, J. 2003, MNRAS, 339, 1256

Komatsu, E., Dunkley, J., Nolta, M. R., et al. 2009, ApJS, 180, 330

Kurki-Suonio, H., Jedamzik, K., \& Mathews, G. J. 1997, ApJ, 479, 31

Lara, J. F., Kajino, T., \& Mathews, G. J. 2006, Phys. Rev. D, 73, 083501

Lepp, S., \& Shull, J. M. 1984, ApJ, 280, 465

Lepp, S., Stancil, P. C., \& Dalgarno, A. 2002, J. Phys. B Atom. Mol. Phys., 35, 57

Loeb, A. 2008 [arXiv:0804.2258]

Longair, M. S. 2008, Galaxy Formation, ed. M. S. Longair (Berlin: Springer)

Mackey, J., Bromm, V., \& Hernquist, L. 2003, ApJ, 586, 1

Maoli, R., Ferrucci, V., Melchiorri, F., Signore, M., \& Tosti, D. 1996, ApJ, 457,

1

Maoli, R., Melchiorri, F., \& Tosti, D. 1994, ApJ, 425, 372

Mizusawa, H., Nishi, R., \& Omukai, K. 2004, PASJ, 56, 487

Nordh, H. L., von Schéele, F., Frisk, U., et al. 2003, A\&A, 402, L21

Okamoto, T. 2008, in New Horizons in Astronomy, ed. A. Frebel, J. R. Maund,

J. Shen, \& M. H. Siegel, ASP Conf. Ser., 393, 111

Olberg, M., Frisk, U., Lecacheux, A., et al. 2003, A\&A, 402, L35

Page 14 of 18 
C. M. Persson et al.: The first spectral line surveys searching for signals from the dark ages

Olofsson, A. O. H., Persson, C. M., Koning, N., et al. 2007, A\&A, 476, 791 Omukai, K., \& Kitayama, T. 2003, ApJ, 599, 738

Papadopoulos, P., Ivison, R., Carilli, C., \& Lewis, G. 2001, Nature, 409, 58 Persson, C. M., Olofsson, A. O. H., Koning, N., et al. 2007, A\&A, 476, 807 Persson, C. M., Olberg, M., . Hjalmarson, A., et al. 2009, A\&A, 494, 637 Prochaska, J. X., Sheffer, Y., Perley, D. A., et al. 2009, ApJ, 691, L27

Puy, D., Alecian, G., Le Bourlot, J., Leorat, J., \& Pineau Des Forets, G. 1993, A\&A, 267, 337

Puy, D., Dubrovich, V., Lipovka, A., Talbi, D., \& Vonlanthen, P. 2007, A\&A 476, 685

Puy, D., \& Signore, M. 1996, A\&A, 305, 371

Puy, D., \& Signore, M. 2007, New Astron. Rev., 51, 411

Rauscher, T., Applegate, J. H., Cowan, J. J., Thielemann, F.-K., \& Wiescher, M. 1994, ApJ, 429, 499

Rohlfs, K., \& Wilson, T. L. 2004, Tools of radio astronomy, ed. K. Rohlfs, \& T. L. Wilson

Rubiño-Martín, J. A., Chluba, J., \& Sunyaev, R. A. 2006, MNRAS, 371, 1939

Rubiño-Martín, J. A., Chluba, J., \& Sunyaev, R. A. 2008, A\&A, 485, 377

Salvaterra, R., Della Valle, M., Campana, S., et al. 2009, Nature, 461, 1258
Santoro, F., \& Shull, J. M. 2006, ApJ, 643, 26

Schleicher, D. R. G., Galli, D., Palla, F., et al. 2008, A\&A, 490, 521

Schneider, R., Ferrara, A., \& Salvaterra, R. 2004, MNRAS, 351, 1379

Sethi, S. K., Subrahmanyan, R., \& Roshi, D. A. 2007, ApJ, 664, 1

Sethi, S. K., Nath, B. B., \& Subramanian, K. 2008, MNRAS, 387, 1589

Signore, M., \& Puy, D. 2009, Eur. Phys. J. C, 59, 117

Silk, J. 1968, ApJ, 151, 459

Smith, B. D., Turk, M. J., Sigurdsson, S., O’Shea, B. W., \& Norman, M. L. 2009, ApJ, 691, 441

Srianand, R., Noterdaeme, P., Ledoux, C., \& Petitjean, P. 2008, A\&A, 482, L39 Stanway, E. R., Bremer, M. N., Davies, L. J. M., et al. 2008, ApJ, 687, L1

Steigman, G. 2007, Ann. Rev. Nuc. Part. Sci., 57, 463

Tanvir, N. R., Fox, D. B., Levan, A. J., et al. 2009, Nature, 461, 1254

Vonlanthen, P., \& Puy, D. 2008, in SF2A-2008: Proceedings of the Annual meeting of the French Society of Astronomy and Astrophysics, ed. C. Charbonnel, F. Combes, \& R. Samadi, 355

Vonlanthen, P., Rauscher, T., Winteler, C., et al. 2009, A\&A, 503, 47

Wise, J. H., \& Abel, T. 2008, ApJ, 685, 40

Woosley, S. E., \& Bloom, J. S. 2006, ARA\&A, 44, 507 


\section{Appendix A: Figures}

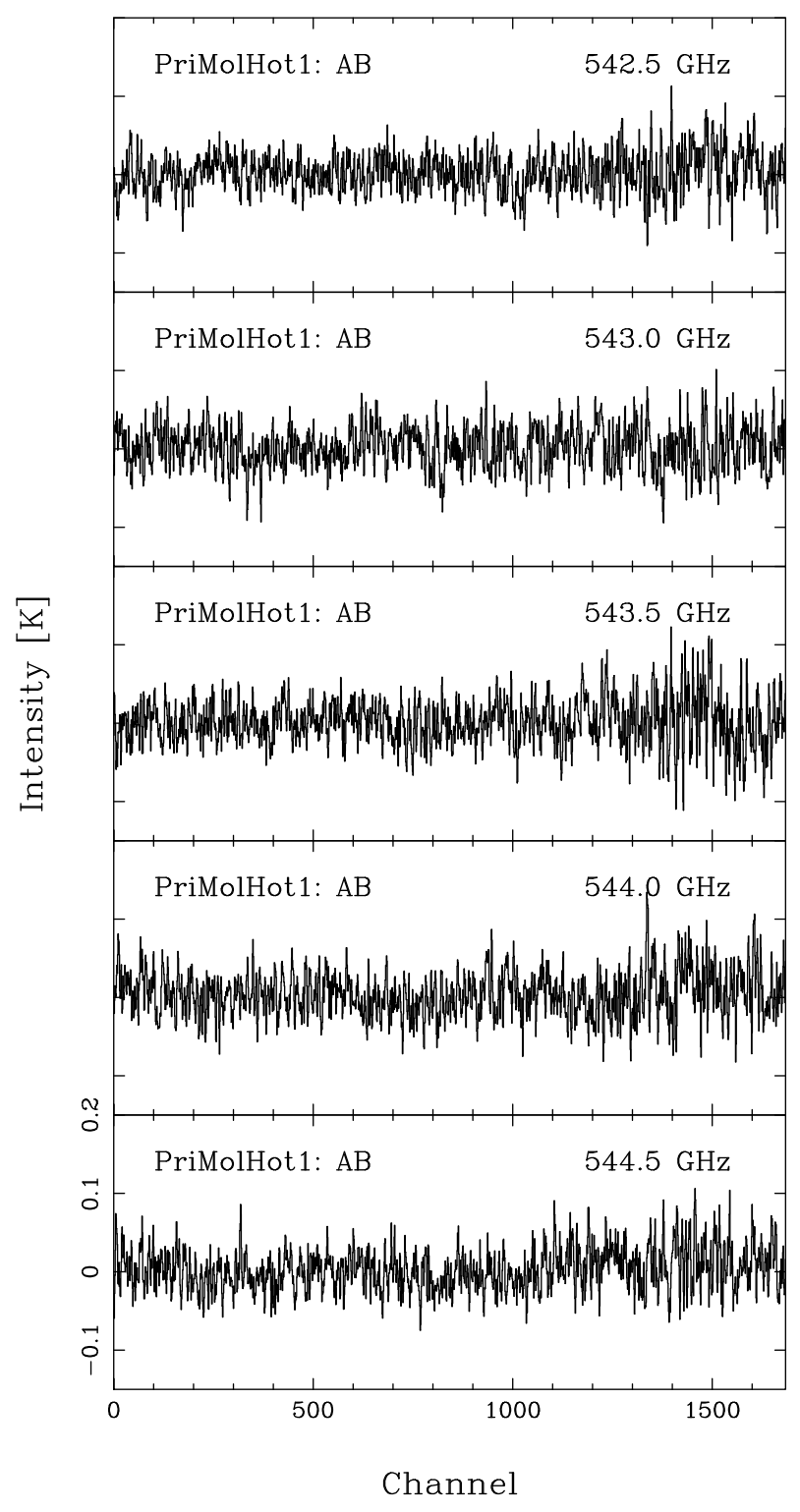

Fig. A.1. Spectra from weekend $1-5$ vs. channels for Position A with B as reference from the 2006/07 survey in original shape and channel spacing $0.62 \mathrm{MHz}$.

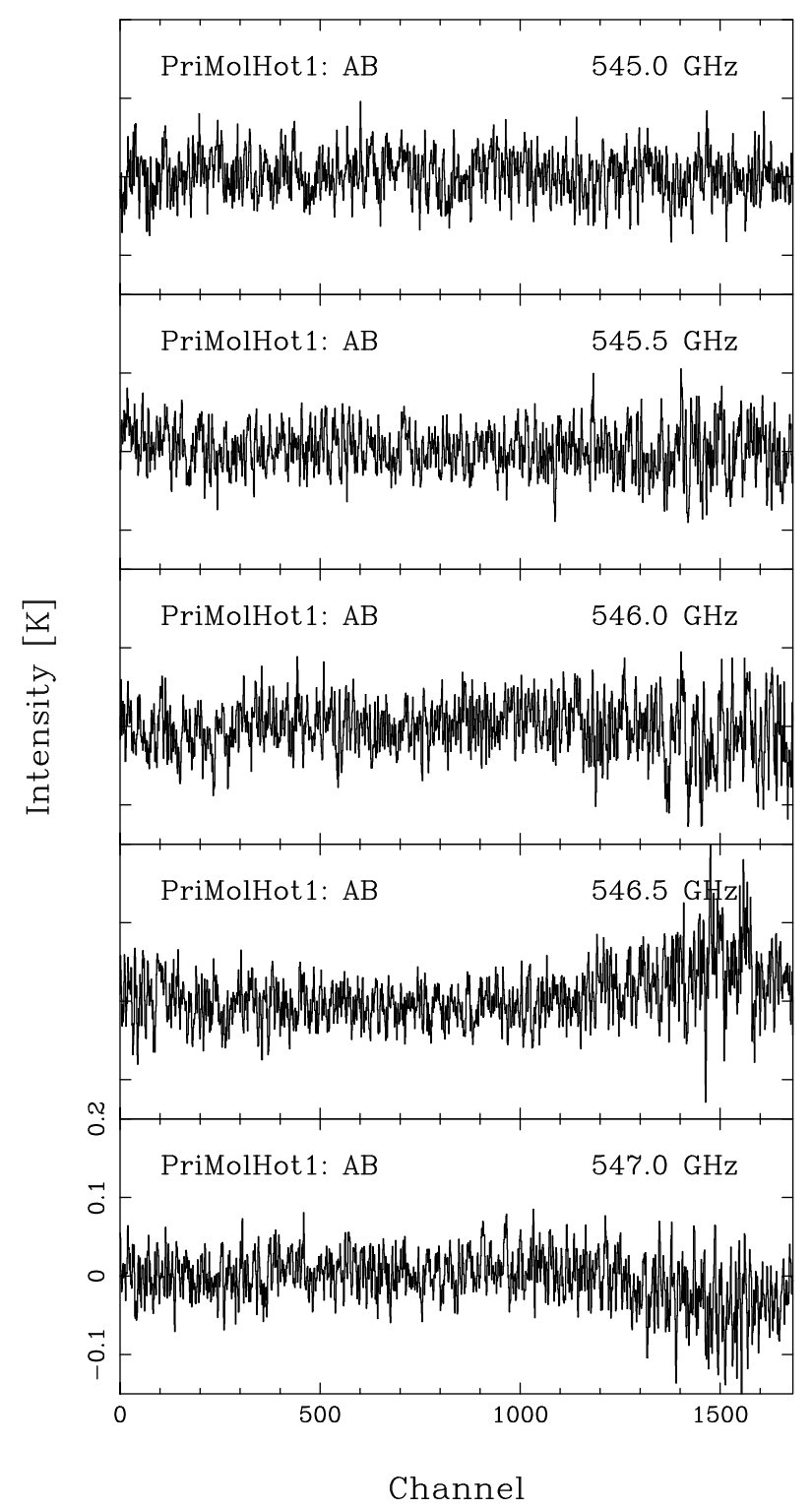

Fig. A.2. Spectra from weekend 6-10 vs. channels for Position A with B as reference from the 2006/07 survey in original shape and channel spacing $0.62 \mathrm{MHz}$.

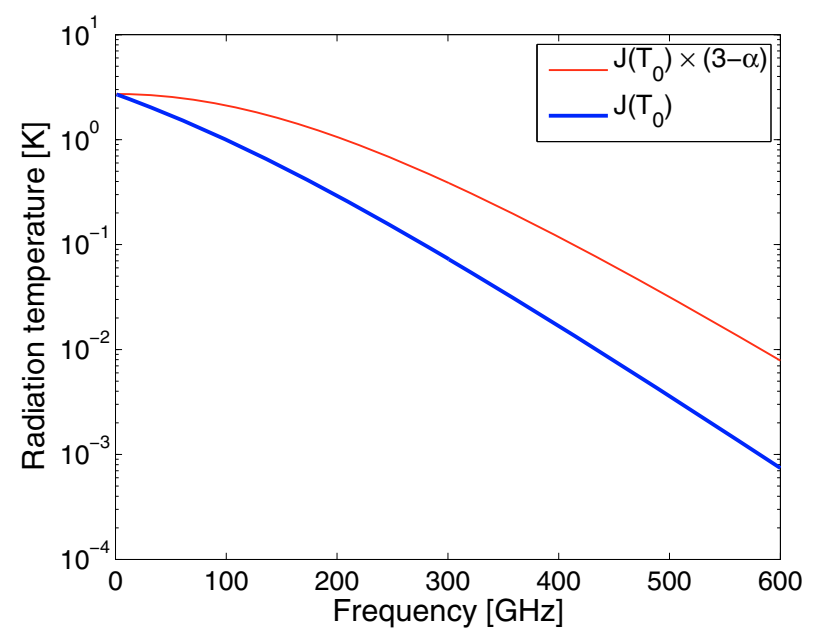

Fig. A.3. The blue thick line shows the radiation temperature $J\left(T_{0}\right)$, and the red thin line shows $J\left(T_{0}\right) \times(3-\alpha)$ as a function of frequency for $T_{0}=2.725 \mathrm{~K}$. 


\section{Appendix B: The turn-around redshift as a function of density fluctuations on the last scattering surface}

Let the unperturbed density at the last scattering surface (LSS) be $\rho_{\mathrm{LSS}}=\rho_{\mathrm{m}_{\mathrm{LSS}}}+\rho_{\Lambda_{\mathrm{LSS}}}+\rho_{\mathrm{rad}_{\mathrm{LSS}}}$, where $\rho_{\mathrm{m}}$ iss is the matter density, $\rho_{\Lambda_{\mathrm{LSS}}}$ is the dark energy density and $\rho_{\mathrm{rad}_{\mathrm{LSS}}}$ the radiation energy density. Since the matter density is very dominant at $\operatorname{LSS} \rho_{\mathrm{LSS}} \approx \rho_{\mathrm{m} \text { LS }}$. This implies that the critical density $\rho_{\text {crit }_{\mathrm{LSS}}}=\rho_{\mathrm{m}_{\mathrm{LSS}}}+\rho_{\Lambda_{\mathrm{LSS}}}+\rho_{\mathrm{rad}_{\mathrm{LSS}}}+\rho_{k_{\mathrm{LSS}}} \approx \rho_{\mathrm{m}_{\mathrm{LSS}}}$ since we assume that the curvature density $\rho_{\mathrm{k}_{\mathrm{LSS}}}$ is zero. The density parameter at the LSS then becomes $\Omega_{\text {LSS }}=\rho_{\text {LSS }} / \rho_{\text {crit }_{L S S}}=1$.

Let a perturbed density at LSS $\rho_{\text {LSS }}^{\prime}$ with the (large) scale $a_{\text {LSS }}$ be defined by

$\rho_{\mathrm{LSS}}^{\prime}=\rho_{\mathrm{LSS}}+\Delta \rho_{\mathrm{LSS}}$,

where $\Delta \rho_{\text {LSS }}$ is the density perturbation. If $\Delta \rho_{\text {LSS }}$ is positive, the turn-around scale $a_{\mathrm{TA}}^{\prime}$ of the perturbed region is (cf. Longair 2008, Eq. (16.2))

$a_{\mathrm{TA}}^{\prime}=a_{\mathrm{LSS}} \frac{\Omega_{\mathrm{LSS}}^{\prime}}{\Omega_{\mathrm{LSS}}^{\prime}-1}$,

where

$\Omega_{\mathrm{LSS}}^{\prime}=\frac{\rho_{\mathrm{LSS}}+\Delta \rho_{\mathrm{LSS}}}{\rho_{\mathrm{LSS}}}=1+\frac{\Delta \rho_{\mathrm{LSS}}}{\rho_{\mathrm{LSS}}}$.

If $\Delta \rho_{\mathrm{LSS}}$ is negative, there is of course no turn-around.

The scale $a_{\mathrm{TA}}$ in the unperturbed region at the turn-around time $t=t_{\mathrm{TA}}$ is then (cf. Longair 2008, Eq. (16.3))

$a_{\mathrm{TA}}=5.55^{1 / 3} \cdot a_{\mathrm{TA}}^{\prime}=5.55^{1 / 3} \cdot a_{\mathrm{LSS}} \frac{\Omega_{\mathrm{LSS}}^{\prime}}{\Omega_{\mathrm{LSS}}^{\prime}-1}$.

Since $a_{\mathrm{TA}}=\left(1+z_{\mathrm{TA}}\right)^{-1}$ and $a_{\mathrm{LSS}}=\left(1+z_{\mathrm{LSS}}\right)^{-1}$ we have

$\left(1+z_{\mathrm{TA}}\right)=\left(1+z_{\mathrm{LSS}}\right) \cdot 5.55^{-1 / 3} \frac{\Delta \rho_{\mathrm{LSS}}}{\rho_{\mathrm{LSS}}+\Delta \rho_{\mathrm{LSS}}}$.

If $\Delta \rho_{\text {LSS }} \ll \rho_{\text {LSS }}$ we have

$\left(1+z_{\mathrm{TA}}\right)=\left(1+z_{\mathrm{LSS}}\right) \cdot 5.55^{-1 / 3} \frac{\Delta \rho_{\mathrm{LSS}}}{\rho_{\mathrm{LSS}}}$.

We may replace $\Delta \rho_{\mathrm{LSS}} / \rho_{\mathrm{LSS}}$ with $\Delta M_{\mathrm{LSS}} / M_{\mathrm{LSS}}$, where $M$ is the mass within the scale $a$, so that

$\left(1+z_{\mathrm{TA}}\right)=\left(1+z_{\mathrm{LSS}}\right) \cdot 5.55^{-1 / 3} \frac{\Delta M_{\mathrm{LSS}}}{M_{\mathrm{LSS}}}$.
Consider now density fluctuations of the Harrison-Zeldovich type (cf. Longair 2008, page 390-392), where

$\sigma_{M}=\left\langle\left(\frac{\Delta M}{M}\right)^{2}\right\rangle^{1 / 2} \sim M^{-2 / 3}$.

Note that this relation may be expressed as

$\left\langle(\Delta M)^{2}\right\rangle^{1 / 2} \sim M^{1 / 3}$.

assuring that black holes are not formed excessively on small or large scales.

Assume now that Eq. (B.8) can be normalised by the observed fluctuations $\sigma_{M_{S}}=10^{-4}$ of the mass $M_{S}=3.72 \times 10^{15} M_{\odot}($ cf. Longair 2008, Eq. (15.13)) within the sound horizon at the last scattering surface where $z_{\text {LSS }}=1090$. Equation (B.8) is then normalised to

$\sigma_{M}=10^{-4}\left(\frac{M_{S}}{M}\right)^{2 / 3}$

Expressing $\Delta M / M$ in Eq. (B.7) in terms of $\xi \cdot \sigma_{M}$, where $\xi$ is a sigma measure, we get

$\left(1+z_{\mathrm{TA}}\right)=\left(1+z_{\mathrm{LSS}}\right) \cdot 5.55^{-1 / 3} \cdot \xi \cdot \sigma_{M}$.

Note that $\xi>0$.

Inserting Eq. (B.10) and $M_{S}$ we get,

$1+z_{\mathrm{TA}}=\left(1+z_{\mathrm{LSS}}\right) \cdot 1.35 \cdot \xi \cdot\left(\frac{10^{9} M_{\odot}}{M}\right)^{2 / 3}$.

This derivation is valid for dark matter. Baryon structure formation, also driven by gravitational forces, has as well been affected by Silk damping (Silk 1968) and sound waves. This complication introduces a minor modification to the power spectrum of the density fluctuations (page 412 in Longair 2008), but approximatively our derivation should hold also for the baryonic matter. More worrisome for the Harrison-Zeldovich scenario are perhaps the findings of massive galaxy clusters at high redshifts, for instance XMMU J2235.3-2557 at $z=1.4$ with a estimated projected mass of the cluster within $1 \mathrm{Mpc}$ of $8.5 \pm 1.7 \times$ $10^{14} M_{\odot}$ (Jee et al. 2009) which according to Eq. (B.12) constitutes a $14 \sigma$ event.

It should perhaps be noted that masses on the order of $10^{8}-10^{9} M_{\odot}$ have turnaround redshifts on the order of $10^{3}-10^{4}$. This means that black holes of this size could form shortly thereafter if the collapse is not halted by induced angular momentum. 


\section{Appendix C: Tables}

Table C.1. The ranges in redshifts covered by our observations for the five lowest rotational lines of $\mathrm{H}_{2}, \mathrm{HD}$, and $\mathrm{HeH}^{+}$.

\begin{tabular}{lrcrccccc}
\hline \hline Species & $\begin{array}{r}\text { Frequency } \\
{[\mathrm{GHz}]}\end{array}$ & $\begin{array}{c}\text { Transitions } \\
J_{u}-J_{l}\end{array}$ & $\begin{array}{r}E_{u} \\
{[\mathrm{~K}]}\end{array}$ & $\begin{array}{c}\text { A-coeff. } \\
{\left[\mathrm{s}^{-1}\right]}\end{array}$ & $\begin{array}{c}\text { AC }[\mathrm{GHz}] \\
486.5-492.0\end{array}$ & $\begin{array}{c}\text { AOS }[\mathrm{GHz}] \\
542.0-547.5\end{array}$ & $\begin{array}{c}\text { AOS }[\mathrm{GHz}] \\
547.0-563.0\end{array}$ & $\begin{array}{c}\mathrm{AC}[\mathrm{GHz}] \\
563.0-578.0\end{array}$ \\
\hline $\mathrm{H}_{2}$ & 10621 & $2-0$ & 510 & $2.94 \times 10^{-11}$ & $z=20.83-20.59$ & $18.60-18.40$ & $18.42-17.87$ & $17.87-17.38$ \\
& 17594 & $3-1$ & 1015 & $4.76 \times 10^{-10}$ & $z=35.16-34.76$ & $31.46-31.14$ & $31.16-30.25$ & $30.25-29.44$ \\
& 24410 & $4-2$ & 1681 & $2.75 \times 10^{-9}$ & $z=49.18-48.61$ & $44.04-43.58$ & $43.63-42.36$ & $42.36-41.23$ \\
& 31011 & $5-3$ & 2503 & $9.83 \times 10^{-9}$ & $z=62.74-62.03$ & $56.22-55.64$ & $55.69-54.08$ & $54.08-52.65$ \\
& 37348 & $6-4$ & 3474 & $2.64 \times 10^{-8}$ & $z=75.77-74.91$ & $67.91-67.22$ & $67.28-65.34$ & $65.34-63.62$ \\
$\mathrm{HD}$ & 2675 & $1-0$ & 128 & $5.32 \times 10^{-8}$ & $z=4.50-4.37$ & $3.94-3.89$ & $3.89-3.75$ & $3.75-3.63$ \\
& 5332 & $2-1$ & 384 & $5.05 \times 10^{-7}$ & $z=9.96-9.84$ & $8.84-8.74$ & $8.75-8.47$ & $8.47-8.22$ \\
& 7952 & $3-2$ & 766 & $1.80 \times 10^{-6}$ & $z=15.35-15.16$ & $13.67-13.52$ & $13.54-13.12$ & $13.12-12.76$ \\
& 10518 & $4-3$ & 1271 & $4.31 \times 10^{-6}$ & $z=20.62-20.38$ & $18.41-18.21$ & $18.23-17.68$ & $17.68-17.20$ \\
& 13015 & $5-4$ & 1895 & $8.35 \times 10^{-6}$ & $z=25.75-25.45$ & $23.01-22.77$ & $22.79-22.12$ & $22.12-21.52$ \\
$\mathrm{HeH}^{+}$ & 2010 & $1-0$ & 96 & 0.109 & $z=3.13-3.09$ & $2.71-2.67$ & $2.67-2.57$ & $2.57-2.48$ \\
& 4009 & $2-1$ & 289 & 1.04 & $z=7.24-7.15$ & $6.40-6.32$ & $6.33-6.12$ & $6.12-5.94$ \\
& 5984 & $3-2$ & 576 & 3.75 & $z=11.30-11.16$ & $10.04-9.93$ & $9.94-9.63$ & $9.63-9.35$ \\
& 7925 & $4-3$ & 956 & 9.14 & $z=15.29-15.11$ & $13.62-13.48$ & $13.49-13.08$ & $13.08-12.71$ \\
& 9821 & $5-4$ & 1428 & 18.1 & $z=19.19-18.96$ & $17.12-16.94$ & $16.95-16.44$ & $16.44-15.99$ \\
\hline
\end{tabular}

Table C.2. Physical sizes corresponding to the Odin beam and the different angular steps in the 2006/07 observations.

\begin{tabular}{rrrrrrrr}
\hline \hline $\begin{array}{r}\text { Angular } \\
\text { size }\end{array}$ & $z=5$ & 10 & 25 & $\begin{array}{c}\text { Redshift } z \\
\text { Physical size }[\mathrm{kpc}]\end{array}$ \\
$\theta\left[^{\prime}\right]$ & & \multicolumn{7}{c}{100} & 200 & 300 \\
\hline $2^{\prime} \cdot 1$ & 820 & 540 & 270 & 150 & 79 & 41 & 28 \\
5 & 2000 & 1300 & 640 & 350 & 190 & 98 & 66 \\
10 & 3900 & 2600 & 1300 & 700 & 380 & 200 & 130 \\
15 & 5900 & 3900 & 1900 & 1060 & 560 & 290 & 200 \\
30 & 12000 & 7700 & 3800 & 2100 & 1100 & 590 & 400 \\
\hline
\end{tabular}

Table C.3. Line widths in the linear phase for a perturbation corresponding to the Odin beam at a number of redshifts.

\begin{tabular}{ccccccc}
\hline \hline $\begin{array}{c}\text { Redshift } \\
z\end{array}$ & $\begin{array}{c}\text { Temp } \\
T \\
{[\mathrm{~K}]}\end{array}$ & $\begin{array}{c}\text { Size }^{a} \\
{[\mathrm{kpc}]}\end{array}$ & $\begin{array}{c}\text { Mass }^{a} \\
M\end{array}$ & $\begin{array}{c}\text { Fract. width } \\
{\left[M_{\odot}\right]}\end{array}$ & $\begin{array}{c}\text { Line width }^{b} \\
\Delta v_{\text {Linear }} \\
{\left[\mathrm{km} \mathrm{s}^{-1}\right]}\end{array}$ & $\begin{array}{c}\text { Width at } 500 \mathrm{GHz} \\
\Delta v \\
[\mathrm{GHz}]]\end{array}$ \\
\hline 10 & 30 & 540 & $4 \times 10^{12}$ & $2 \times 10^{-3}$ & 720 & 1.3 \\
20 & 57 & 320 & $6 \times 10^{12}$ & $4 \times 10^{-3}$ & 1100 & 2.1 \\
50 & 139 & 150 & $9 \times 10^{12}$ & $7 \times 10^{-3}$ & 2000 & 3.7 \\
75 & 207 & 100 & $9 \times 10^{12}$ & $8 \times 10^{-3}$ & 2500 & 4.5 \\
100 & 275 & 79 & $1 \times 10^{13}$ & $1 \times 10^{-2}$ & 2900 & 5.3 \\
150 & 411 & 54 & $1 \times 10^{13}$ & $1 \times 10^{-2}$ & 3600 & 6.6 \\
200 & 547 & 41 & $1 \times 10^{13}$ & $1 \times 10^{-2}$ & 4200 & 7.7 \\
\hline
\end{tabular}

Notes. ${ }^{(a)}$ For an angular size of the Odin beam, 2 .1 calculated with Eqs. $(5)-(7) ;{ }^{(b)}$ the line width during the linear evolution, Eq. (8). 